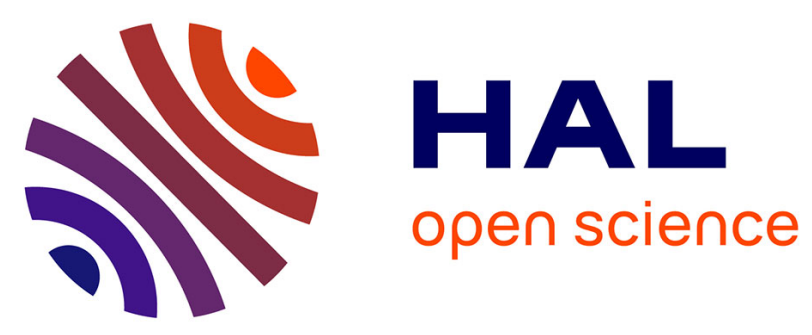

\title{
Ocular inflammation induces trigeminal pain, peripheral and central neuroinflammatory mechanisms
}

Pierre-Serge Launay, Elodie Reboussin, Hong Liang, Karima Kessal, David

Godefroy, William Rostene, José-Alain Sahel, Christophe Baudouin, Stéphane

Melik Parsadaniantz, Annabelle Reaux Le Goazigo

\section{To cite this version:}

Pierre-Serge Launay, Elodie Reboussin, Hong Liang, Karima Kessal, David Godefroy, et al.. Ocular inflammation induces trigeminal pain, peripheral and central neuroinflammatory mechanisms. Neurobiology of Disease, 2016, 88, pp.16-28. 10.1016/j.nbd.2015.12.017 . hal-01258703

\section{HAL Id: hal-01258703 https: / hal.sorbonne-universite.fr/hal-01258703}

Submitted on 19 Jan 2016

HAL is a multi-disciplinary open access archive for the deposit and dissemination of scientific research documents, whether they are published or not. The documents may come from teaching and research institutions in France or abroad, or from public or private research centers.
L'archive ouverte pluridisciplinaire HAL, est destinée au dépôt et à la diffusion de documents scientifiques de niveau recherche, publiés ou non, émanant des établissements d'enseignement et de recherche français ou étrangers, des laboratoires publics ou privés. 


\section{Ocular inflammation induces trigeminal pain, peripheral and central}

\section{neuroinflammatory mechanisms}

LAUNAY Pierre-Serge $e^{1,2,3 *}$, REBOUSSIN Elodie ${ }^{1,2,3 *}$ LIANG Hong ${ }^{1,2,3,4}$, KESSAL Karima $^{1,2,3}$, David GODEFROY ${ }^{1,2,3}$, William ROSTENE ${ }^{1,2,3}$, SAHEL Jose-Alain ${ }^{1,2,3,4}$, BAUDOUIN Christophe $e^{1,2,3,4}$, MELIK PARSADANIANTZ Stéphane $e^{1,2,3}$, REAUX LE GOAZIGO Annabelle $e^{1,2,3}$.

* contribute equally to the work

1 INSERM, U968, Paris, F-75012, France

2 Sorbonne Universités, Université UPMC, Paris 06, UM 80, Institut de la Vision, 75012 Paris, France

3 CNRS, UMR 7210, Paris, F-75012, France

4 Centre Hospitalier National d'Ophtalmologie des Quinze-Vingts, Paris, F-75012, France

\section{* Correspondence:}

Annabelle REAUX-LE GOAZIGO

Institut de la Vision

UMR S 968 Inserm/ UPMC/ CNRS 7210

17, rue Moreau 75012 Paris

Phone :+ 33 1-53-46-25-72

annabelle.reaux@inserm.fr

\section{Running title:}

Chronic ocular injury induces neuroinflammation in the sensory trigeminal complex

Keywords: benzalkonium chloride, corneal neurons, ocular pain, neuroinflammation, trigeminal complex, facial motor nucleus. 


\section{ABSTRACT}

Ocular surface diseases are among the most frequent ocular pathologies, with prevalence ranging from $20 \%$ of the general population. In addition, ocular pain following corneal injury is frequently observed in clinic. The aim of the study was to characterize the peripheral and central neuroinflammatory process in the trigeminal pathways in response to cornea alteration induced by chronic topical instillations of $0.2 \%$ benzalkonium chloride (BAC) in male C57BL/6J mice. In vitro BAC induced neurotoxicity and increases neuronal (FOS, ATF3) and pro-inflammatory (IL-6) markers in primary mouse trigeminal ganglion culture. BAC-treated mice exhibited 7 days after the treatment reduced aqueous tear production and increased inflammatory cell infiltration in the cornea. Hypertonic saline-evoked eye wipe behavior was enhanced in BAC-treated animals that exhibited increased FOS, ATF3 and Iba1 immunoreactivity in the trigeminal ganglion. Ocular inflammation is associated with a significant increase in IL-6 and TNF- $\alpha$ mRNA expression in the trigeminal ganglion. We reported a strong increase in FOS and Ibal positive cells in particular in the sensory trigeminal complex at the ipsilateral interpolaris/caudalis $(\mathrm{Vi} / \mathrm{Vc})$ transition and $\mathrm{Vc} / \mathrm{upper}$ cervical cord $(\mathrm{Vc} / \mathrm{C} 1)$ regions. In addition, activated microglial cells were tightly wrapped around activated FOS neurons in both regions and phosphorylated p38 mitogen-activated protein kinase was markedly enhanced specifically in microglial cells during ocular inflammation. Similar data were obtained in the facial motor nucleus. These neuroanatomical data correlated with the increase in mRNA expression of pro-inflammatory (TNF- $\alpha$, IL-6, CCL2) and neuronal (FOS and ATF3) markers. Interestingly, the suppression of corneal inflammation 10 days following the end of BAC treatment resulted in a marked attenuation of peripheral and central changes observed in pathological conditions.

This study provides the first demonstration that corneal inflammation induces activation of neurons and microglial p38 MAPK pathway within sensory trigeminal complex. These results 
suggest that this altered activity in intracellular signaling caused by ocular inflammation might play a priming role in the central sensitization of ocular related brainstem circuits, which represents a significant factor in ocular pain development. 


\section{Highlights}

BAC induced corneal nerve injury, inflammation and ocular pain

Ocular inflammation is associated with activation of FOS and Iba1 in the trigeminal ganglia

Chronic ocular inflammation increased peripheral and central pro-inflammatory mediators

Microglial p38 activation occurred in sensory trigeminal complex

We reported central plastic changes under ocular inflammation 


\section{INTRODUCTION}

Ocular surface diseases are among the most frequent ocular pathologies, with prevalence ranging from $20 \%$ of the general population. Chronic ocular pain, frequently observed in clinic, has gained recognition as a public health problem given its prevalence, morbidity, and social cost implications.

The cornea receives the densest nociceptive innervation of the body, which is exclusively from small-fiber neurons (Belmonte et al., 2004; Muller et al., 1997; Rozsa and Beuerman, 1982). The cellular bodies of the corneal neurons are located in the dorsomedial portion of the ophthalmic region of trigeminal ganglion (TG) (Launay et al., 2015; Marfurt and Del Toro, 1987; Marfurt et al., 1989) and represent only $1.3 \%$ of the total population of the TG cells (Felipe et al., 1999). The central axons of corneal sensory neurons terminate in two regions of the sensory trigeminal complex $(\mathrm{V})$ : the trigeminal subnucleus interpolaris/caudalis $(\mathrm{Vi} / \mathrm{Vc})$ transition and the subnucleus caudalis/upper cervical cord $(\mathrm{Vc} / \mathrm{C} 1)$ junction regions (Marfurt and Del Toro, 1987; Meng and Bereiter, 1996; Strassman and Vos, 1993). Neuroanatomical and electrophysiological studies have demonstrated that second order neurons activated after a noxious stimulation of the cornea exhibited a high-regionalized distribution in the Vi/Vc and Vc/C1 regions (Kurose and Meng, 2013; Stapleton et al., 2013). Several lines of evidence suggest that the $\mathrm{Vc} / \mathrm{C} 1$ region integrates irritation and noxious stimuli of the cornea, whereas the $\mathrm{Vi} / \mathrm{Vc}$ region and facial motor nucleus seem to be more dedicated to the control of lacrimation and the blinking reflex (Kurose and Meng, 2013; Stapleton et al., 2013).

Numerous preclinical models have been developed to evaluate the consequences of corneal injury or dry eye disease on the activation of the trigeminal pathways: alkali burn cornea (Ferrari et al., 2014), models for tear deficient dry eye disease after lachrymal gland excision (Katagiri et al., 2015; Rahman et al., 2015) or after chronic instillation of benzalkonium chloride (BAC), a bactericidal preservative in ophthalmic preparation (Liang et al., 2012; 
Liang et al., 2008; Lin et al., 2011; Sarkar et al., 2012).

A recent study has reported that corneal alkali-burn induced a strong immune cell infiltration in both the cornea and TG (Ferrari et al., 2014). This immune cell infiltration is correlated with strong overexpression of pro-inflammatory markers. Additionally, an electrophysiological study has demonstrated that persistent dry eye caused sensitization of ocular-responsive neurons in the sensory trigeminal complex (Rahman et al., 2015). To date there is no evidence about the inflammatory mechanisms and the changes in brain microglia that occurred in the sensory trigeminal complex and the facial motor nucleus during chronic corneal inflammation.

The aim of the study was to determine the effects of chronic corneal inflammation induced by seven days of BAC instillations on the neuroinflammatory mechanisms that occurred in the trigeminal pathways ( $\mathrm{TG}, \mathrm{Vi} / \mathrm{Vc}$ and $\mathrm{Vc} / \mathrm{C} 1$ regions and the facial motor nucleus). In vivo, at the corneal level, we correlated the effect of chronic BAC instillation on nerve terminal integrity with the expression of inflammatory cytokines/chemokines. Furthermore, our animal model provides an excellent opportunity to evaluate the relationship between corneal injury/sensitivity and the peripheral and central changes in Fos, Iba1 and p38 MAPK activation that may play a role in central sensitization within the sensory trigeminal complex. 


\section{MATERIAL AND METHODS}

\subsection{Animals}

Adult male C57BL/6 mice (30 g; Janvier Labs, Le Genest Saint Isle, France) were maintained under controlled conditions $\left(22 \pm 1{ }^{\circ} \mathrm{C}, 60 \pm 10 \%\right.$ relative humidity, $12 / 12 \mathrm{~h}$ light/dark cycle, food and water ad libitum). All procedures were in strict accordance with the guidelines for the care and use of experimental animals approved by the French National Institute of Medical Research (INSERM), national and international laws and with policies for the use of animals in Research in Vision and Ophthalmology (European Communities Council Directive 86/609/EEC, Authorization No. 75-1235 Granted to A. R-LG). Animals were weighed before treatment (Day 0, D0) and at the end of the experiments, Day 7 (D7).

\subsection{Drugs}

The solution containing $0.2 \%$ Benzalkonium Chloride (BAC) was provided by Santen pharmaceutical (Osaka, Japan). This iso-osmolar solution (osmolarity of $270 \mathrm{mOsM}$ ) corresponds to a molecular weight of $354 \mathrm{~g} \cdot \mathrm{mol}^{-1}$ and a concentration of $5.649 \mathrm{mM}$.

\subsection{Primary trigeminal ganglion culture}

TGs were aseptically removed from mice and placed in TG medium on ice. TGs were pooled in $2 \mathrm{~mL}$ of DMEM medium containing collagenase A (Sigma-Aldrich) and incubated at $37^{\circ} \mathrm{C}$ for $90 \mathrm{~min}$ and then in Trypsin /EDTA- DNase I solution (Sigma-Aldrich) for $15 \mathrm{~min}$ at $37^{\circ} \mathrm{C}$. Neurobasal A medium (Gibco) was then added and TG cells were mechanically dissociated using a Pasteur pipette. Dissociated cells were pelleted by centrifugation, the collagenase-containing supernatant discarded and cells resuspended in Neurobasal A 
supplemented with 5\% horse serum (Gibco), 2\% B-27 supplement (Gibco), L -glutamine (0.1 $\mathrm{mg} / \mathrm{ml}$, Sigma-Aldrich), $45 \%$ glucose, penicillin (100 U/ml), and streptomycin (100 L g/ml). Cells were then filtered using a $70 \mu \mathrm{m}$ filter and were distributed into two 24 -well culture plates that had been coated with poly-D-lysine/laminin. Cultures were incubated in a $37^{\circ} \mathrm{C}$ tissue culture incubator $\left(5 \% \mathrm{CO}_{2}, 95 \%\right.$ humidity). The medium was changed the following day and completed with $\mathrm{AraC}$ at $10 \mu \mathrm{M}$ to prevent glial cell proliferation. The medium was changed $24 \mathrm{~h}$ before BAC treatment. After 9 days of culture, TG cells were either treated with $0.005 \%$ or $0.0005 \%$ BAC solutions for $6 \mathrm{~h}$ or received no treatment. After treatment, cells were gently washed with $1 \mathrm{x}$ PBS and processed for immunohistochemistry or RT-qPCR analysis. We carefully checked that the number of the neurons per area were the same in all condition.

\subsection{Topical instillation}

Mice were gently restrained and $10 \mu \mathrm{L}$ of $0.2 \%$ BAC or $1 x$ PBS (274 mOsM) solutions were applied with a micropipette to the left eye. Mice received the drug two times per day separated by a 4 hours interval. Eyes were held open for 20 seconds to prevent aggressive blinking during application of the drug, which may cause variability in the ocular surface contact time of the drug. For in vivo experiments related to recovery after topical BAC treatment, mice were treated twice a day for 7 days with a $0.2 \%$ BAC solution and followed for 10 days after stopping the BAC instillation.

\subsection{In vivo characterization of the preclinical animal model of ocular pain}

\subsubsection{Measurement of spontaneous aqueous tear production}

Were mice lightly anaesthetized with a $300 \mu \mathrm{L}$ mixture of Ketamine $1000 \mathrm{U}$ (100 mg/kg body weight $)$ and xylazine (10 mg/kg body weight, Virbac, France) injected intraperitoneally. Tear 
production was measured with the phenol red thread test (Zone- Quick; Lacrimedics, Eastsound, WA). The threads were held with jeweler forceps and placed in the lateral canthus of the conjunctival fornix of the eye for 30 seconds as previously described (Lin et al., 2011). Tear production was monitored on D0 and D7. The thread is yellow in color (acidic) and when exposed to tears, it changes to a light red color. After $30 \mathrm{~s}$, the tear distance (in millimeters) is determined using a scale.

\subsubsection{Biomicroscopic evaluation of the cornea}

Fluorescein staining of the corneal epithelium was used to evaluate corneal integrity as previously described (Liang et al., 2012). On D0 (before the instillation) and D7 (1 h after the last instillation), ocular surfaces were evaluated by the fluorescein test as described below.

A drop of $1 \%$ fluorescein sodium (Fluoresceine Faure 0.5\%; Novartis France, RueilMalmaison, France) was instilled in the left eye of anaesthetized mice. The ocular surface was photographed using a SteREAO Lumar microscope (Zeiss). The biomicroscope was calibrated for control mice before acquisitions in BAC-treated mice.

\subsubsection{In vivo confocal microscopy}

A laser-scanning in vivo confocal microscopy (IVCM, Heidelberg Retina Tomography (HRT) II/Rostock Cornea Module (RCM; Heidelberg Engineering GmbH, Heidelberg, Germany) was used to examine the entire cornea of anaesthetized mice. The first layer of superficial epithelium was considered as $0 \mu \mathrm{m}$, and the depth cornea was determined manually, 0-30 $\mu \mathrm{m}$, $30-40 \mu \mathrm{m}$, the stroma $40-140 \mu \mathrm{m}$ and the endothelium, $140-150 \mu \mathrm{m}$. A minimum of 200 serial TIFF images (400 x $400 \mu \mathrm{m})$ was acquired per animal.

\subsubsection{Behavioral study: Eye Wiping Test}


The eye wiping test (Farazifard et al., 2005) was used to evaluate corneal sensitivity at D0 (before the treatment to obtain basal threshold) and D7. The same experimenter performed all experiments in a test room close to the colony room, to minimize stress. Behavioral experiments were carried out in single-blind conditions (the investigator was blinded to the treatment group). For the adaptation period, animals were placed in an individual Plexiglas chamber (where the tests occurred) for ten minutes each day for 4 days. A $10 \mu \mathrm{L}$ drop of $2 \mathrm{M}$ $\mathrm{NaCl}$ solution was then applied to the treated eye, the animal placed in an individual cage and the investigator counted the number of wipes for a period of 30s. Normal facial grooming behavior was not included.

\subsubsection{Weight measurement}

In order to evaluate whether ocular pain lead to alterations in weight gain, animals were weighed at D0 and D7.

\section{$2.6 R T-q P C R$ analysis}

For in vivo experiments, mice were deeply anaesthetized with a $300 \mu \mathrm{L}$ mixture of Ketamine $1000 \mathrm{U}$ (100 mg/kg body weight) and xylazine (10 mg/kg body weight, Virbac, France) injected intraperitoneally. Animals were then perfused with cold $10 \mathrm{~mL} 0.9 \% \mathrm{NaCl}$ solution and the cornea, brainstem and TGs were carefully dissected out as described below. For the cornea, the eyeball was extracted and placed in a petri dish containing cold 1x PBS solution placed on ice. Corneas were meticulously dissected out and placed in liquid azote. For ipsilateral TGs, ganglia were dissected out (the mandibular branch was removed with a scalpel blade) and placed in liquid azote. Ipsilateral trigeminal brainstem complex was dissected and placed in liquid azote until the extraction procedure. 
RNAs were extracted from TG cells, cornea and TGs using the XS Macherey-Nagel NucleoSpin RNA extraction kit and Macherey-Nagel NucleoSpin RNA extraction kit was used for the brainstem, according to the manufacturer's protocol.

Reverse transcription was performed with 660 ng of RNA using the High Capacity cDNA Reverse Transcription (Applied Biosystems) according to the manufacturer's instructions. Finally, cDNA were diluted in DNAse/RNAse free water to a final concentration of $5 \mathrm{ng} . \mu \mathrm{L}^{-1}$. Real-time quantitative PCR was performed with $25 \mathrm{ng}$ of cDNA added to a $15 \mu \mathrm{L}$ solution of Applied Biosystems mastermix (TaqMan Universal PCR Master Mix) and primers to a final volume of $20 \mu \mathrm{L}$. All primers and reagents were purchased from Applied Biosystems. The primers used were ATF3 (Mm00476032.m1), CCL2 (Mm99999056.m1), CX3CR1 (Mm00438354.m1), FOS (Mm00487425.m1), GFAP (Mm01253033.m1), IL1b (Mm00434228.m1), IL6 (Mm00446190.m1) ITGAM (Mm00434455.m1), TNF- $\alpha$ (Mm99999068.m1), TRPV1 (Mm01246302.m1). Specific mRNA levels were calculated after normalization of the results for each sample with those for GADPH mRNA. The data are presented as relative mRNA units with respect to control values (expressed as a fold-over PBS value).

\subsection{Immunohistological studies}

\subsubsection{Tissue preparation}

Two hours after the last instillation of BAC, a $300 \mu \mathrm{L}$ mixture of Ketamine $1000 \mathrm{U}$ (100 $\mathrm{mg} / \mathrm{kg}$ body weight) and xylazine (10 mg/kg body weight, Virbac, France) was injected intraperitoneally to deeply anesthetized animals. Eyes were dissected out just before the perfusion. Mice were perfused via the ascending aorta with $5 \mathrm{~mL}$ of $0.9 \% \mathrm{NaCl}$ solution followed by $30 \mathrm{~mL}$ of $4 \%$ paraformaldehyde solution. After fixation, brain, trigeminal ganglia, and eyes were carefully dissected out and post-fixed $48 \mathrm{~h}$ in the same fixative. Free- 
floating sections $(40 \mu \mathrm{m})$ of the trigeminal subnucleus complex (trigeminal nucleus interpolaris to the spinal cord level C1) were performed using a vibratome (Leica Microsystems, Germany). The TGs were placed in $10 \%$ sucrose solution in 1x PBS (overnight), then in a $30 \%$ sucrose solution before freezing at $-20^{\circ} \mathrm{C}$ in $7.5 \%$ gelatin and $10 \%$ sucrose. Cryostat sections $(14 \mu \mathrm{m})$ were performed for TGs and eyes, then mounted on Superfrost slides and keep at $-20^{\circ} \mathrm{C}$ until use.

\subsubsection{Immunofluorescence labeling}

After three washes in 1x PBS, brainstem and TG sections were placed in a blocking solution containing 3\% Normal Horse Serum (NHS) and 0.1\% triton X-100 (only for free floating sections), for $2 \mathrm{~h}$, then incubated with primary antibody at $4^{\circ} \mathrm{C}$ for $48 \mathrm{~h}$ (floating sections) or $24 \mathrm{~h}$ for cryostat sections.

The primary antibodies used in this study were rabbit anti-ATF3 (Sigma-Aldrich: HPA001562, 1:500), rabbit anti-FOS (Santa Cruz Biotechnology: A2113, 1:500), mouse anti GFAP (Sigma-Aldrich: G3893, 1:500), goat-anti Iba1 (Abcam: ab5076, 1:500) and rabbit anti-pP38 (Cell Signaling: 4511S, 1:500). After incubation of the primary antibody, all other procedures were performed at room temperature (RT). ATF3, FOS and p-P38 were amplified using biotin-conjugated horse anti-rabbit antibody (1:500; Vector Laboratories) for $1 \mathrm{~h}$ and finally revealed by incubation with streptavidin-Alexa Fluor 488/555 (1:500; Invitrogen). Iba1 was revealed using Alexa Fluor 594-conjugated donkey anti-goat (1:500; Invitrogen) and GFAP was revealed with Alexa Fluor 594-conjugated donkey anti-mouse (1:500; Invitrogen) for $1 \mathrm{~h}$ respectively.

TG sections were processed using the avidin-biotin peroxidase method (Vectastain ABC Kit, Vector Laboratories) for ATF3 and Iba1 immunoreactivity. TG sections were incubated with $3 \% \mathrm{H}_{2} \mathrm{O}_{2}$ solution for $20 \mathrm{~min}$. Sections were rinsed three times in $1 \mathrm{x}$ PBS then incubated in 
$3 \%$ NHS for $2 \mathrm{~h}$ at RT before incubation with primary antibodies overnight at $4{ }^{\circ} \mathrm{C}$. Sections were rinsed three times in 1x PBS the incubated for $1 \mathrm{~h}$ at RT with the secondary biotinylated anti-rabbit $\operatorname{IgG}$ (1/500: Vector). Sections were rinsed three times in 1x PBS, incubated in a solution containing the Avidin Biotin Peroxidase Complex (Elite, Vector) for $1 \mathrm{~h}$, then rinsed again three times with $1 \mathrm{x}$ PBS. The peroxidase reaction was developed by incubating the sections in 0.05\% 3,3'-diaminobenzidine (DAB; Sigma Aldrich) containing 0.003\% hydrogen peroxide in $0.05 \mathrm{M}$ Tris $\mathrm{HCl}$ buffer $\mathrm{pH} 7.6$ for 5 to $10 \mathrm{~min}$. Sections were rinsed in 1x PBS and mounted onto gelatin-coated slides, dried and mounted with Eukitt mounting medium (Kindler).

Fluoro-Jade stain (Fluoro-Jade C Staining Kit, TR-100-FJ, Biosensis) was used to label degenerating neurons. Briefly, cryostat TG sections and free-floating brainstem sections from PBS and BAC-treated animals were mounted on slides and treated according to the manufacturer's recommendations: $5 \mathrm{~min}$ in sodium hydroxide/70 \% ethanol, $20 \mathrm{~min}$ in potassium permanganate solution and $20 \mathrm{~min}$ in the Fluoro-Jade $\mathrm{C}$ staining solution. After staining, slides were washed in distilled water, dried at RT then mounted with Eukitt. For histological analysis of the cornea, sections were stained with hematoxylin-eosin according to the manufacturer's recommendations.

\subsection{Microscopy and quantification of immunohistochemical labeling}

\subsubsection{Microscopic devices}

Cells and tissue sections were examined either with a Zeiss M1 epifluorescence microscope or an Olympus FV1000 laser-scanning confocal microscope. The epifluorescence microscope (Axio ImagerM1; Carl Zeiss) was equipped with a digital camera (Axio Cam HRC; Carl Zeiss) and image acquisition software (Zen; Carl Zeiss). The Olympus FV1000 confocal microscope was equipped with an Argon (488 nm) ion laser and laser diodes (405 and 559 
$\mathrm{nm})$. Images were acquired sequentially, line-by-line, in order to reduce excitation and emission crosstalk. Step size was defined according to the Nyquist-Shannon sampling theorem (1024*1024 pixels). Objectives used were a PlanApo (10/0.40 NA) or PlanApoN (60/1.42 NA, oil immersion) objective lens (Olympus). Tissue sections were also scanned with the Nanozoomer 2.0-HT Digital slide scanner (C9600, Hamamatsu Photonics), equipped with the 3-CCD TDI camera (Hamamatsu Photonics). TIFF images were obtained. The microscope was calibrated for control mice before acquisitions in BAC-treated mice.

2.8.2 In vitro quantification of the surface occupied by neuronal nerve fibers in primary TG culture

TG cells were fixed for $15 \mathrm{~min}$ in $4 \%$ paraformaldehyde in PBS then rinsed three times with 1x PBS. Nonspecific binding sites were blocked by $3 \%$ NHS for $1 \mathrm{~h}$ at room temperature in PBS containing $0.1 \%$ Triton $\mathrm{X}-100$. Cells were then incubated with mouse anti-Milli-Mark ${ }^{\mathrm{TM}}$ Pan Neuronal Marker (1:1000, Merk Millipore) containing 1\% NGS for $1 \mathrm{~h}$. The cells were then rinsed three times with $1.0 \mathrm{~mL}$ of PBS. Subsequently, the cells were incubated with Alexa Fluor 488-conjugated donkey anti-mouse antibody (1:1000, Invitrogen) for $1 \mathrm{~h}$ and washed three times with PBS. The cells were then mounted and stored at $4^{\circ} \mathrm{C}$ until observation. Cells treated without primary antibody served as controls.

Quantitative analysis of the surface covered by primary TGs fibers in culture was performed. Briefly, epifluorescent images were acquired for each condition. Images were saved as TIFF files and processed using ImageJ (version 1.48). Using the threshold function of ImageJ, objects of interest from the surrounding background could be distinguished. The total surface occupied by immunoreactive structures (i.e. total stained pixels) above the threshold was estimated within a standard area. Results were expressed as the mean \pm SEM of ten different culture fields per condition. 


\subsubsection{D Reconstruction of confocal images}

For 3D reconstruction in the trigeminal complex, 35 stacks of $0.54 \mu \mathrm{m}$ (corresponding to a depth of $17 \mu \mathrm{m}$ ) were performed in PBS- and BAC-treated animals using the same confocal settings. TIFF images were analyzed with the Imaris 7.6.1 software (Bitplane, Zurich, Switzerland) in order to generate a movie. The background subtraction option was set at 5 for both FOS and Iba1. Using the surface area detail option of the Imaris software, the FOS and Iba1 settings were calibrated at 1.00 and 0.34 , respectively. Movies from 3D confocal images were performed using the $40 \mathrm{x}$ objective and $2 \mathrm{x}$ numerical zoom. Videos were set at 400 frames per second.

\subsection{Statistical analysis}

All data are presented as mean \pm SEM. Data were evaluated by one-way analysis of variance with the non-parametric ANOVA test using the GraphPad Prism 4.0 statistical software. The level of significance was set at $P<0.05$. 


\section{RESULTS}

\section{BAC induces neurotoxicity and pro-inflammatory markers in primary cultures of mouse trigeminal ganglion.}

To study the impact of BAC solution on primary mouse trigeminal ganglion cultures, TG cells were initially exposed for $6 \mathrm{~h}$ to two concentrations of BAC $\left(5.10^{-4} \%\right.$ and $5.10^{-5} \%$, corresponding to a concentration of $14.12 \mu \mathrm{M}$ and $1.41 \mu \mathrm{M}$, respectively), and the TG cells were stained with Pan Neuronal Marker to quantify the nerve fibers in culture (Fig 1 A-C). A dose dependent neurotoxicity effect of BAC was observed as illustrated in Fig 1 A-C. The quantification of area covered by immunoreactive nerve fibers confirmed a significant decrease in nerve fibers: $21 \%$ and $70 \%$ for BAC solution at concentrations of $5.10^{-5} \%$ and $5.10^{-4} \%$, respectively (Fig. 1D). RT-qPCR analysis of TG cells showed an increase in the expression of neuronal makers (ATF3 and FOS) and for the pro-inflammatory marker IL6: FOS $(\mathrm{X} 1.28 \pm 0.06, \mathrm{P}<0.05 ; \mathrm{X} 2.13 \pm 0.26, \mathrm{P}<0.01)$, ATF3 $(\mathrm{X} 1.55 \pm 0.11, \mathrm{P}<0.01 ; \mathrm{X} 3.05 \pm$ $0.46, \mathrm{P}<0.01)$ and IL-6 $(\mathrm{X} 1.25 \pm 0.10, \mathrm{P}<0.05 ; \mathrm{X} 2.97 \pm 0.26, \mathrm{P}<0.01$; with respect to control conditions) for BAC solutions at concentrations of $5.10^{-5} \%$ and $5.10^{-4} \%$, respectively (Fig. 1E). We carefully checked that the number of the neurons per area were the same in all condition after BAC treatment (data not show).

\section{BAC induces dry eye and ocular inflammation}

We then determined the effects of $0.2 \%$ BAC treatment on ocular surface. Mice were instilled twice a day for 7 days. Corneal integrity was evaluated with the fluorescein test at D0 and D7. No difference was observed between the two (control and BAC) groups of animals at D0 (not shown). At D7, fluorescein staining of the cornea was greater with $0.2 \%$ BAC than with PBS (Fig 2A). Hematoxylin-eosin (HE) staining of corneal sections from BAC animals confirmed 
the destruction of the superficial corneal epithelium and the presence of numerous cells (probably immune cells) in the stroma (Fig. 2B, white arrows).

In vivo confocal microscopy (IVCM) (Fig. 2C) was used to image the different corneal layers: epithelium, sub-basal plexus, stroma and endothelium. Control mice exhibited a healthy corneal epithelium with pavimentous cells (Fig. 2D), a sub-basal plexus containing numerous thin nerve fibers, a stroma with classical square cells (keratocytes), a large trunk nerves (Fig. 2D, yellow arrows) and an endothelium with regular dark reflective cells (Fig. 2D). BAC treatment induced important injury in the ocular surface characterized by a complete destruction of the superficial epithelium (not detected under IVCM), and immune cell infiltration (numerous bright and reflective immune cells) in the sub-basal plexus and the stroma (Fig. 2D, red arrows). Furthermore, corneal nerves were less abundant in both layers as compared to control cornea (Fig. 2D). BAC treatment did not alter the endothelium (Fig. 2D). Spontaneous tear volume was identical between both groups at D0 (not shown). Tear production was significantly reduced in the ipsilateral eyes of 7 days BAC-treated mice (1.33 $\pm 0.16 \mathrm{~mm}$ compared with control: $4.83 \pm 0.49 \mathrm{~mm}$; P<0.05) (Fig. 2E). RT-qPCR analysis of the cornea revealed increased pro-inflammatory cytokine gene expression (TNF- $\alpha$ : X4.89 \pm

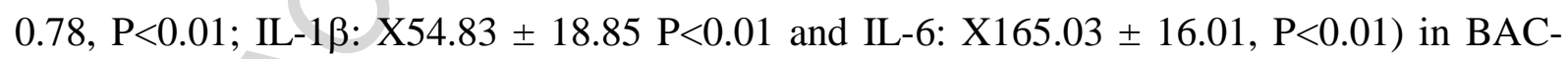
treated cornea, with respect with control animals (Fig. 2F).

\section{Eye wipe behavior}

Sensitivity of the cornea to hypertonic saline was assessed by quantifying eye wipe behavior in response to the application of $2 \mathrm{M}$ saline to the eyes in separate groups of animals. At D0, no significant difference was observed between the two groups $(9.40 \pm 0.62$ wipes versus $10.00 \pm 0.61$ wipes for the PBS- and BAC-treated groups, respectively). At D7, application of $2 \mathrm{M} \mathrm{NaCl}$ solution increased eye wipe behavior in BAC-treated animals $(24.00 \pm 0.98$ wipes 
versus $9.70 \pm 0.37$ wipes in PBS treated mice, respectively, $\mathrm{P}<0.001$ ) (Fig. 3A). We did not observe spontaneous wiping in BAC treated eyes before hypertonic saline stimulation. Figure 3B illustrates a typical spontaneous eye-blink behavior observed at D7; the treated eye remains closed (Fig. 3B). In addition, we observed a decreased (79\%) body weight gain in BAC-treated animals, relative to control at D7 $(0.34 \pm 0.11$ versus $1.64 \pm 0.09 \mathrm{~g} ; \mathrm{P}<0.001)$ (Fig. 3C).

Activation of primary order neurons and pro-inflammatory markers in the ophthalmic branch of the trigeminal ganglion.

At D7, we compared the ATF3 and FOS immunostaining on ipsilateral TG from both groups of mice. Chronic BAC treatment increased ATF3 and FOS positive nuclei in primary sensory neurons compared to PBS-treated mice (Fig. 4A-B, arrows). In addition, increased Iba1-ir cells were detected in the ipsilateral TG of BAC-treated mice (Fig. 4C, arrows). Nonetheless, no difference was observed in GFAP staining (a marker of satellite ganglion cell) in both groups of animals (Fig. 4D, arrows). Interestingly, RT-qPCR analysis performed on ipsilateral TG revealed that BAC treatment induced a significant increase in pro-inflammatory markers (TNF- $\alpha$ : X1.38 $\pm 0.10, \mathrm{P}<0.01$ and IL-6: X1.26 $\pm 0.07, \mathrm{P}<0.01$ ), with respect with control animals (Fig. 4E). However, no significant change was observed for FOS, ATF3, CCL2, IL$1 \beta$ and GFAP mRNA (Fig. 4E).

Neuronal activation and microglial reorganization in the sensory trigeminal complex and facial motor nucleus.

We then investigated neuronal activation (using FOS staining) and microglial cells (Iba1 staining) in the ipsilateral and contralateral sensory trigeminal complex $(\mathrm{Vi} / \mathrm{Vc}$ and $\mathrm{Vc} / \mathrm{C} 1$ transition regions), known to contain primary afferent projections from the cornea. In PBS- 
treated mice, there was no difference in FOS staining between ipsilateral (Fig. 5A,C) and contralateral (not shown) sides of the sensory trigeminal complex. BAC-treated animals exhibited increased FOS- positive neurons in the ventrolateral and a dorsolateral part of the ipsilateral $\mathrm{Vi} / \mathrm{Vc}$ and $\mathrm{Vc} / \mathrm{C} 1$ regions, respectively (Fig. 5A,C, left panels). Interestingly, a low FOS activation was also observed in the contralateral $\mathrm{Vi} / \mathrm{Vc}$ and $\mathrm{Vc} / \mathrm{C} 1$ regions. In $\mathrm{BAC}$ treated mice, FOS was accompanied by a central inflammatory response in the brainstem. While control mice exhibited resting microglial cells with thin ramifications and small cellular bodies (Fig. 5A-D, middle panels), BAC-treated mice exhibited activated (amoeboid morphology with a large soma and short processes) microglia in both $\mathrm{Vi} / \mathrm{Vc}$ and $\mathrm{Vc} / \mathrm{C} 1$ regions (Fig. 5A-D, middle panels). Activated microglia was evident in both ipsilateral and contralateral $\mathrm{Vi} / \mathrm{Vc}$ and $\mathrm{Vc} / \mathrm{C} 1$ trigeminal regions; however, the density and intensity of FOS and Iba1 positive cells were stronger in the ipsilateral regions (Fig. 5A,C). Interestingly, a local reorganization of activated Iba1-positive microglia was observed; microglial cells were tightly wrapped around activated FOS-positive neurons in both regions (Fig. 5A,C, right panels).

3D reconstitutions of double labeled brain sections illustrates these morphological changes of microglial cells in contact with or in close apposition to activated neurons in the ipsilateral $\mathrm{Vi} / \mathrm{Vc}$ region (see Movie 1 and 2). In parallel we observed an elevated activation (phosphorylation) of p38 MAPK in the ipsilateral trigeminal nucleus of animals exhibiting ocular pain. Furthermore, p-p38 immunoreactivity was restricted to microglial (Iba1-positive) cells in the ventral ipsilateral $\mathrm{Vi} / \mathrm{Vc}$ and $\mathrm{Vc} / \mathrm{C} 1$ transitions. Only scattered p-p38 positive microglial cells were detected in the contralateral $\mathrm{Vi} / \mathrm{Vc}$ and $\mathrm{Vc} / \mathrm{C} 1$ regions, whereas labeling of p-p38 was not observed in control mice (Fig. 5B,D).

We then used a FluoroJade C staining (a highly specific marker for the detection of neurodegenerative neurons) to evaluate whether ocular pain induced neuronal cell death in the 
TG and trigeminal nucleus. There was no neuronal damage in BAC-treated mice, compared to the positive control tissues (substantia nigra) obtained from the 1-methyl-4-phenyl-1,2,3,6tetrahydropyridine (MPTP) mouse model of Parkinson disease (not shown).

The facial motor nucleus is responsible for the early response of the corneal blink reflex observed after corneal stimulation. Similarly to what was observed in the sensory trigeminal complex, ipsilateral facial motor nucleus of BAC-treated animals showed increased FOSpositive neurons and Iba1-positive microglia as compared to that of PBS-treated mice (Fig. 6A). Furthermore, the upregulation of FOS immunoreactivity was also seen to a lower extent in the contralateral facial motor nucleus of BAC-treated animals.

All Iba-1 positive microglia exhibited an 'activated form'. As previously observed for Vi/Vc and $\mathrm{Vc} / \mathrm{C} 1$ regions, activated Iba1-positive microglia were tightly wrapped around and/or attached to activated FOS neurons in both ipsilateral and contralateral facial motor nuclei of BAC-treated animals. Moreover, we observed that activation of p38 (p-p38) is restricted into Iba1-positive microglia in BAC-treated mice, while p-p38 immunoreactivity was not observed in control mice (Fig. 6B). In our study, we also observed that unilateral ocular inflammation produced some increase in p-P38 immunoreactivity in the contralateral facial motor nucleus (Fig. 6B).

\section{Activation of proinflammatory markers in the sensory trigeminal complex.}

In addition to evaluate neuronal and microglial cell changes, we tested whether peripheral corneal inflammation could have an impact on proinflammatory gene expression in the ipsilateral sensory trigeminal complex. To assess this, we preformed RT-qPCR analysis. We observed that animals suffering from ocular pain demonstrated an upregulation of both neuronal and pro-inflammatory markers in the sensory trigeminal complex: FOS (X1.25 \pm 0.07, P<0.01), ATF3 (X2.19 $\pm 0.38, \mathrm{P}<0.001)$, TNF- $\alpha(\mathrm{X} 1.33 \pm 0.07, \mathrm{P}<0.001)$, CCL2 
$(\mathrm{X} 1.79 \pm 0.19, \mathrm{P}<0.001), \mathrm{IL}-6(\mathrm{X} 1.12 \pm 0.04, \mathrm{P}<0.05)$ mRNA, with respect with control animals (Fig. 7).

In light of the observation that chronic BAC treatment induced corneal inflammation, peripheral and central sensitizations, these processes should be reduced following termination of BAC treatment. To prove this, we examined whether such inhibition is observed 10 days after terminating BAC treatment. Observations 10 days after withdrawal of BAC treatment showed that mice did not anymore exhibit an eye-blink behavior (not shown). Slit lamp examination showed reduced fluorescein staining in these BAC withdrawal mice, compared to 7 days BAC-treated mice (Fig. 8A). In vivo confocal microscopy images confirmed corneal wound re-epithelialization and reduced immune cell infiltration in the stroma, as well as corneal nerve regeneration (Fig. 8B). Hematoxylin-eosin staining of corneal sections from BAC withdrawal mice confirmed a re-epithelialization of the corneal epithelium and less immune cells in the stroma (Fig. 8C, black arrows). BAC withdrawal mice still exhibited a lower weight gain at D17 (1.33 $\pm 0.09 \mathrm{~g}$ versus $3.14 \pm 0.15 \mathrm{~g})$ : this reduction $(-57 \%)$ is less important than that observed in BAC-treated animals (-79\%) (Fig. 8D). Interestingly, microscopic analysis of FOS and Iba1 staining in the ophthalmic region of the TG showed a decreased activation of primary sensory neurons and a decreased cell infiltration in BAC withdrawal mice, compared to BAC-treated mice (Fig. 8E). In the trigeminal complex, a clear decrease in neuronal activation (FOS immunoreactivity) and inflammation (Iba1 immunoreactivity) in the sensory trigeminal complex at the level of $\mathrm{Vi} / \mathrm{Vc}$ (Fig. 8F), Vc/C1 (Fig. 8G) and facial motor nucleus (Fig. 8G) can be noticed. More importantly, the specific reorganization of activated microglial cells previously observed after BAC treatment disappeared in all regions of interest. 


\section{DISCUSSION}

In the present study we have investigated the peripheral and central neuroinflammation that occurred in trigeminal pain-related pathways during ocular inflammation. We used a toxicological approach that involves repeated topical instillations of $0.2 \% \mathrm{BAC}$ solution for one week. This treatment induced a strong corneal inflammation and decreased spontaneous tear production. In addition, we also observed a sustained ocular pain-related behavior, enhanced activation of primary sensory neurons, immune cell infiltration, and proinflammatory marker expression in the TG of BAC-treated mice. We reported a highly specific spatial reorganization of activated microglia cells wrapped around second orderactivated neurons and an increase in pro-inflammatory gene expression markers in the sensory trigeminal complex (Fig. 9). Interestingly, all of these peripheral and central cellular changes disappeared 10 days after terminating BAC treatment, when ocular inflammation disappeared. Here we showed that ocular inflammation is characterized by severe alterations in the corneal epithelium and a deep infiltration of inflammatory cells in the sub-basal plexus and stroma. This inflammatory phenotype was correlated with an overexpression of pro-inflammatory cytokine mRNA (i.e IL-1 $\beta$, IL-6 and TNF- $\alpha$ ) in the cornea. Previous studies using corneal wound models highlighted the toxic inflammatory effect of BAC on the ocular surface (Liang et al., 2012; Lin et al., 2011; Sarkar et al., 2012).

In vivo confocal microscopy reveal that corneal nerve fibers are drastically damaged in BACtreated animals supporting our in vitro data on TG primary cells and a previous study showing that topical BAC induced corneal neurotoxicity in Thy1-YFP+ mice, which exhibited a disappearance of corneal nerve fluorescence (Sarkar et al., 2012).

Corneal inflammation plays a central role in the generation of nociceptor sensitization. Proinflammatory cytokines released during inflammation may activate polymodal nociceptor nerve terminals. This peripheral nociceptor sensitization was reported to be responsible of the 
distinct quality and persistence of pain arising from inflamed tissues (Belmonte et al., 2015; Melik Parsadaniantz et al., 2015). The eye wipe behavior test, classically used to evaluate acute ocular nociceptive sensitization, confirms higher corneal sensitivity in BAC-treated mice. Our behavioral data are in contrast with a study that reported decreased ocular sensitivity in rabbit eyes treated with a $0.02 \%$ BAC solution (Chen et al., 2013), but is comparable to a recent study evaluating corneal sensitivity in a rat model of dry eye disease (Meng et al., 2015). The reasons for these differences are unclear, but may be related to the fact that the authors performed corneal mechanical stimulation versus a chemical test used in the present study. Nonetheless, data concerning the decreased spontaneous production of tears and corneal alterations are in agreement with those previously published in rodents (Chen et al., 2011; Lin et al., 2011; Xiong et al., 2008).

Here, we have shown that chronic BAC treatment significantly increases mRNA expression of pro-inflammatory (IL-6 and TNF- $\alpha$ ) and immune cell infiltration (Iba1 immunoreactive cells) markers in the ispilateral TG. The inflammation observed in the TG corroborates with that observed in the alkali-burn corneal pain mouse model (Ferrari et al., 2014). The authors reported a strong infiltration of immune cells associated with an overexpression of proinflammatory markers IL-1 $\beta$, and TNF- $\alpha$. It is well accepted that released proinflammatory mediators from neurons and satellite glia within sensory ganglia increased excitability of primary sensory neurons via autocrine and/or paracrine mechanisms which may contribute to chronic pain (Ma et al., 2003; Melik Parsadaniantz et al., 2015; Takeda et al., 2009). These mechanisms, recently reviewed (Belmonte et al., 2015; Melik Parsadaniantz et al., 2015), may explain the higher corneal sensitivity observed in BAC-treated mice.

Corneal neurons have been well characterized by their electrophysiological properties, neuropeptide content, size and localization in the TG (Bron et al., 2014; Felipe et al., 1999; Launay et al., 2015; Nakamura et al., 2007). Further, we have analyzed the population of 
primary sensory neurons activated by using FOS staining, which is a relevant index to evaluate neuronal activation (Mitsikostas and Sanchez del Rio, 2001). We report a higher number of FOS positive cells in the ophthalmic portion of the TG during ocular inflammation. These data can be correlated to the overexpression of the proto-oncogene c-Jun protein (another index of cellular activation) in corneal nociceptive neurons in the corneal alkali burn model (De Felipe and Belmonte, 1999). We have also shown increased ATF3 staining (that represents a reliable marker of ongoing neuronal damage) in the ophthalmic region of the TG during ocular inflammation. The in vivo increase in ATF3 staining is in accordance with its enhanced mRNA expression observed in vitro on TG cells treated with BAC. However, we did not observe a significative increase in ATF3 mRNA; this could be explained by the fact that corneal neurons only represent $2 \%$ of the total neuronal population of the TG (De Felipe and Belmonte, 1999). Moreover, considering the negative Fluoro jade C staining in the TG (not shown), in our experimental condition chronic ocular inflammation does not induce any apoptotic signal in primary nociceptive neurons. In addition, we cannot exclude the possibility that the increased ATF3 staining may also represent a signalling pathway that participates in corneal nerve regeneration by increasing the intrinsic growth state of injured neurons (for review see (Patodia and Raivich, 2012)). Ten days after terminating BAC treatment, mice recovered a corneal innervation comparable to control mice. These data are in agreement with the results obtained by Sarkar et al, which highlighted the ability of damaged corneal nerves to recover after withdrawal of BAC treatment (Sarkar et al., 2012).

Following chemical or thermal corneal stimulation (Chang et al., 2010; Hirata and Oshinsky, 2012; Meng and Bereiter, 1996), the sensory trigeminal complex ( $\mathrm{Vi} / \mathrm{Vc}$, and $\mathrm{Vc} / \mathrm{C} 1$ transitions) showed an increase of FOS cells. Furthermore, neurons activated in the Vc/C1 region seem to play a more crucial role in ocular pain (Tashiro et al., 2010). Here we found a higher activation of neurons in the $\mathrm{Vc} / \mathrm{C} 1$ than the $\mathrm{Vi} / \mathrm{Vc}$ region confirming the hypothesis 
that sustained ocular inflammation activates pain-related neural pathways. The observed activation of neurons in the ipsilateral $\mathrm{Vi} / \mathrm{Vc}$ region is consistent with the fact that this region (which can directly or indirectly activate motoneurons of the facial motor nucleus) participates in the central regulation of lacrimation and the blinking reflex (Hirata and Oshinsky, 2012). This is in agreement with the decreased spontaneous tear production and the persistent eye blinking observed in our BAC-treated mice.

Increasing evidence suggests an important role of microglia in the genesis of persistent pain, by releasing proinflammatory cytokines. To our knowledge, this is the first evidence suggesting that corneal nerve injury induces a substantial morphologic change of microglia, characterized by activated microglial cells wrapped around second order-activated neurons in the sensory trigeminal complex.

MAPKs play critical roles in neuronal plasticity and inflammatory responses and MAPK activation is correlated with most if not all persistent pain conditions. The activated (phosphorylated) form of the p38 MAPK (p-p38) plays an important role in the development and maintenance of nerve injury-induced pain hypersensitivity (Ji and Suter, 2007). Here, we demonstrate for the first time that corneal nerve injury elicits activation of p38 in the sensory trigeminal complex, which is restricted to the activated microglia and is associated with increased synthesis of pro-inflammatory mediators (IL-6, TNF- $\alpha$, CCL2). Thus, inflammatory cytokines, probably released from microglia in a p38 MAPK dependent manner, may participate in the generation of central sensitization.

There is increasing evidence that unilateral nerve injury evokes contralateral responses (Cheng et al., 2014; Garcia-Larrea and Peyron, 2013). The release of proinflammatory mediators, such as IL-1 $\beta$, TNF- $\alpha$ and Substance $\mathrm{P}$ has been proposed to mediate these bilateral effects (Koltzenburg et al, 2009; Kleinschnitz et al 2005). A recent study has reported changes in the contralateral side of the inflamed eye after corneal alkali burn (Ferrari 
et al., 2014). The authors have found that proinflammatory cytokines (IL-1 $\beta$, TNF- $\alpha$ ) and substance $\mathrm{P}$ were increased not only in the TG ipsilateral to the injury, but also in the contralateral one. Similarly we reported neuronal and microglial activation on both ipsilateral and contralateral brainstem after chronic BAC treatment, though the contralateral responses being always lower than in the ipsilateral side.

Therefore, the sensory trigeminal complex undergoes marked plastic changes (reversed after termination of BAC treatment) in pathological conditions, leading to activation of projection neurons. Trigeminal second order neurons are not only a critical relay center in trigeminal nociceptive transmission but also an important player in the development and maintenance of central sensitization. The substantial morphologic changes of microglia observed in the $\mathrm{Vi} / \mathrm{Vc}$ and $\mathrm{Vc} / \mathrm{C} 1$ regions extend to the ipsilateral facial motor nucleus. We reported a clear activation of p38 in microglia and FOS in this nucleus. These observations have never been reported and could be related to ocular pain. Microglial cell activation (together with increased lymphocyte cell infiltration and TNF- $\alpha$, IL-1 $\beta$ and IFN $\gamma$ mRNA gene expression) has only been observed after facial nerve axotomy in the facial motor nucleus (Dauer et al., 2011; Shokouhi et al., 2010).

In conclusion, our study provides novel insights into the central morphological changes of microglia during sustained ocular inflammation. We showed that chronic ocular inflammation induces persistent activation of FOS, Iba1 and p38 within pain-related neural pathways. This altered activity in intracellular signaling is likely to be linked to the pain related behavior that is seen in our animal model and might explain the persistent pain experienced by patients chronically suffering from ocular pain (such as in dry eye disease). These central neuroinflammatory mechanisms that occur in ocular pain should be taken into account when developing new therapeutic approaches to alleviate chronic ocular pain. 


\section{Acknowledgements}

This study was supported by the Agence Nationale pour la Recherche Grant R06282DS, Université Pierre and Marie Curie (Paris VI), the Institut National de la Santé et de la Recherche Médicale LABEX (ANR-10-LABX-65) and AFER. The authors thank R. N. Cooper for editorial assistance with this manuscript. We thank Anais Sapienza and the staff of the animal house facilities at the Institut de la Vision for their precious help. 


\section{FIGURE LEGEND}

Fig. 1: In vitro neurotoxicity caused by BAC treatment in primary trigeminal ganglion culture. (A-C) Microscopic images showing changes in morphological nerve fibers stained with Pan neuronal marker after being exposed to low concentrations of BAC solution for $6 \mathrm{~h}$. Scale bar $=200 \mu \mathrm{m}$. (D) Histograms showing the percentage of the area covered by immunoreactive nerve fibers in different culture conditions. (E) Changes of ATF3, FOS, IL$1 \beta$ and IL-6 mRNA gene expression after exposure to BAC solution. Results are expressed as mean \pm SEM. $\mathrm{n}=5$ for each condition. Symbols for statistics $* \mathrm{p}<0.01$ vs. control cells $* * \mathrm{P}<$ 0.005 vs. control cells.

Fig. 2: In vivo and ex vivo evaluation of corneal integrity. (A) Corneal fluorescein staining at D7. Note the strong ulceration of the BAC-treated cornea. (B) Hematoxylin-eosin (HE) staining on a corneal section showing cell infiltration (white arrows) in the stroma after BAC treatment. Scale bar: $50 \mu \mathrm{m}$. (C) Non invasive imaging of the cornea with the in vivo confocal microscopy device. (D) In vivo confocal microscope (IVCM) images showing the epithelium $(0-30 \mu \mathrm{m})$, sub-basal plexus $(30-40 \mu \mathrm{m})$, stroma $(40-140 \mu \mathrm{m})$ and endothelium $(140-150$ $\mu \mathrm{m})$ from PBS- and BAC-treated animals. Note the decreased numbers of corneal nerves (yellow arrow) and increased numbers of inflammatory cells (red arrows). Scale bar $=100$ $\mu \mathrm{m}$. (E) Aqueous tear production evaluated by Schirmer test. (F) Quantification of TNF- $\alpha$, IL6 and IL-1 $\beta$ mRNA by RT-qPCR at D7 in the cornea. All data are expressed as means \pm SEM. $* * \mathrm{P}<0.01$ and $* * * \mathrm{P}<0.001$ relative to $\mathrm{PBS}$ mice.

Fig. 3: Eye wiping test and cumulative weight gain in PBS- and BAC-treated animals.

(A) Number of eye wipes in PBS- and BAC-treated mice. BAC significantly increased ocular sensitivity ( $\mathrm{P}<0.001, \mathrm{n}=10$ each group). (B) A typical image of a BAC-treated mouse 
exhibiting a closed eye-blink behavior (white arrow). (C) BAC-treated animals exhibited a reduced weight gain at D7 compared to control mice. The values are expressed as mean \pm SEM. $* * * \mathrm{P}<0.001$ relative to PBS mice ( $\mathrm{n}=20-23$ per group).

Fig. 4: Bright-field and epifluorescent images of ATF3, FOS, Iba1, and GFAP immunolabeling (A-D) and FOS, ATF3, CCL2, IL-6, IL-1ß, TNF- $\alpha$ and GFAP mRNA gene expression (E) in the TG. 7 days of chronic BAC treatment increased ATF3 (A), FOS (B) and Iba1 (C) immunostaining in the ophthalmic branch of the TG compared to the TG of PBS-treated mice. No difference in GFAP immunorecativity was observed between the two groups of animals (D). Arrows indicate immunoreactive cells. Scale bar $=25 \mu \mathrm{m}$. RT-qPCR analysis of neuronal and pro- inflammatory cell markers in the TG (E). Results are expressed as mean \pm SEM. $* * \mathrm{P}<0.01$ relative to $\mathrm{PBS}$ mice $(\mathrm{n}=15-18$ per group).

Fig. 5: Epifluorescent images of double-immunolabeling of Iba1 with FOS (A-C) and pp38 (B-D) in the ipsilateral and contralateral Vi/Ve (A-B) and Vc/C1 (C-D) transition regions of PBS and BAC-treated mice. Schematic drawing from Paxinos atlas illustrating the stereotaxic coordinates of $\mathrm{Vi} / \mathrm{Vc}$ and $\mathrm{Vc} / \mathrm{C} 1$ regions. Red inset indicates the region of interest. Microscopic images revealed that chronic BAC treatment increased FOS, Iba1 and phosphorylated p38 positive cells in the ipsilateral (ipsi) region compared to PBS-treated mice. Unilateral chronic instillations also produced cellular activation in contralateral (contra) regions. White arrows indicate colocalization of markers. Scale bar $=100 \mu \mathrm{m}$.

Fig. 6: Epifluorescent images of double-immunolabeling of Iba1 with FOS (A) and p-p38 (B) in the ipsilateral and contralateral facial motor nucleus of PBS- and BAC-treated mice. Schematic illustration from Paxinos atlas of the stereotaxic coordinates of the facial 
motor nucleus. Representative images of Iba1, FOS, and p-p38 immunoreactivity in the ipsilateral and contralateral facial motor nucleus of PBS and BAC treated animals. Chronic BAC treatment increased FOS immunoreactivity in the ipsilateral (ipsi) and contralateral (contra) facial motor nuclei. Note that ipsilateral and contralateral activations of p38 strictly occurred in Iba1-positive microglial cells. White arrows indicate colocalization of markers. Scale bars $=200 \mu \mathrm{m}(\mathrm{A})$ and $100 \mu \mathrm{m}(\mathrm{B})$.

Fig. 7: Expression of FOS, ATF3, CCL2, IL-6, TNF- $\alpha$, CX3CR1, ITGAM mRNA in the ipsilateral sensory trigeminal complex of PBS- and BAC-treated mice. Animals treated with topical BAC for 7 days exhibit significant upregulation of FOS, ATF3, CCL2, IL-6 and TNF- $\alpha$. Data are expressed as mean \pm SEM. $* * P<0.005$ relative to PBS-treated mice $(n=15-$ 18 per group).

Fig. 8: Ex vivo and in vivo comparisons of the cornea, TG and sensory trigeminal complex from mice that recovered 10 days after BAC treatment withdrawal and mice treated with BAC for 7 days. Fluorescein staining (A) in both groups of mice. Note the significantly decreased intensity of fluorescein staining in BAC withdrawal mice. IVCM images (B) showing the epithelium, sub-basal plexus, stroma and endothelium from BACtreated animals and BAC withdrawal mice. Comparison of weight gain in the treated groups (D). Immunostaining of FOS, Iba1 in the TG (E), $\mathrm{Vi} / \mathrm{Vc}(\mathrm{F})$ and $\mathrm{Vc} / \mathrm{C} 1(\mathrm{G})$ and in the facial motor nucleus $(\mathrm{H})$. Note that immunostaining for FOS and inflammatory markers (Iba1 and pp38) were reduced in all structures of BAC withdrawal mice. Scale bars $=25 \mu \mathrm{m}(\mathrm{C}), 25 \mu \mathrm{m}$ (E), $200 \mu \mathrm{m}(\mathrm{F}-\mathrm{H})$. 
Fig. 9: Schematic representation of the peripheral and central neuroinflammatory processes subject to corneal injury-induced ocular pain. Corneal injury is characterized by immune cell infiltration and pro-inflammatory gene overexpression (TNF- $\alpha$, IL-6, IL-1 $)$. Corneal nerve lesion and inflammation lead to activation of primary sensory neurons (ATF3, FOS markers) and inflammation in the ophthalmic region of the TG (increased Iba1 immunoreactivity and TNF- $\alpha$ and IL-6 mRNA expression). This neuroinflammation is transferred to the sensory trigeminal complex ( $\mathrm{Vi} / \mathrm{Vc}, \mathrm{Vc} / \mathrm{C} 1$ and facial motor nucleus) characterized by activated microglial cells (Iba1/p-p38 labeled cells wrapped around activated second-order FOS positive neurons) and increased pro-inflammatory mediators. Activation of oculomotor neurons (FOS) in the facial motor nucleus results from a direct (blue second order neuron projections) or an indirect (grey interneuron) projection. Activated microglia may also participate in the stimulation of oculomotor neurons leading to a blinking behavior observed in BAC-treated mice.

Movie 1: Resting microglial cells in the ventral Vi/Ve transition region of control animals.

The movie was built using the Imaris 7.6.1 software (Bitplane, Zurich, Switzerland). Individual TIFF images are overlays of the recorded green and red fluorescence channels showing FOS positive second order neurons (Alexa 488) and Iba1 positive cells (Alexa 594), respectively. Note: Background subtraction option was set at 5. Using the surface area detail option, the setting for FOS and Iba1 staining was calibrated at 1.00 and 0.34 , respectively. 35 stacks of $0.54 \mu \mathrm{m}$ using $40 \mathrm{x}$ objective and $2 \times$ numerical zoom Time: 16s, frames: 400, format: avi. 
Movie 2: Activation and reorganization of microglial cells in the ventral $\mathrm{Vi} / \mathrm{Vc}$ transition region following chronic $\mathrm{BAC}$ treatment.

The movie was built using the Imaris 7.6.1 software (Bitplane, Zurich, Switzerland). Individual TIFF images are overlays of the recorded green and red fluorescence channels showing FOS positive second order neurons (Alexa 488) and Iba1 positive cells (Alexa 594), respectively. Note that activated microglia were tightly wrapped around activated FOSpositive neurons in the brainstem. 35 stacks of $0.54 \mu \mathrm{m}$. Time: $16 \mathrm{~s}$, frames: 400 , format: avi. 


\section{References}

Belmonte, C., Aracil, A., Acosta, M.C., Luna, C., Gallar, J., 2004, Nerves and sensations from the eye surface. Ocul Surf, 2, 248-253

Belmonte, C., Acosta, M.C., Merayo-Lloves, J., Gallar, J., 2015, What Causes Eye Pain? Curr Ophthalmol Rep, 3, 111-121

Bron, R., Wood, R.J., Brock, J.A., Ivanusic, J.J., 2014, Piezo2 expression in corneal afferent neurons. J Comp Neurol, 522, 2967-2979

Chang, Z., Okamoto, K., Tashiro, A., Bereiter, D.A., 2010, Ultraviolet irradiation of the eye and Fos-positive neurons induced in trigeminal brainstem after intravitreal or ocular surface transient receptor potential vanilloid 1 activation. Neuroscience, 170, 678-685

Cheng, C.F., Cheng J.K., Chen, C.Y., Lien, C.C., Chu D., Wang, S.Y., Tsaur, M.L., 2014, Mirror-image pain is mediated by nerve growth factor produced from tumor necrosis factor alpha-activated satellite glia after peripheral nerve injury. Pain, 155, 906-920

Chen, W., Li, Z., Hu, J., Zhang, Z., Chen, L., Chen, Y., Liu, Z., 2011, Corneal alternations induced by topical application of benzalkonium chloride in rabbit. PLoS One, 6, e26103

Chen, W., Zhang, Z., Hu, J., Xie, H., Pan, J., Dong, N., Liu, Z., 2013, Changes in rabbit corneal innervation induced by the topical application of benzalkonium chloride. Cornea, 32, $1599-1606$

Dauer, D.J., Huang, Z., Ha, G.K., Kim, J., Khosrowzadeh, D., Petitto, J.M., 2011, Age and facial nerve axotomy-induced $\mathrm{T}$ cell trafficking: relation to microglial and motor neuron status. Brain Behav Immun, 25, 77-82

De Felipe, C., Belmonte, C., 1999, c-Jun expression after axotomy of corneal trigeminal ganglion neurons is dependent on the site of injury. Eur J Neurosci, 11, 899-906

Farazifard, R., Safarpour, F., Sheibani, V., Javan, M., 2005, Eye-wiping test: a sensitive animal model for acute trigeminal pain studies. Brain Res Brain Res Protoc, 16, 44-49 
Felipe, C.D., Gonzalez, G.G., Gallar, J., Belmonte, C., 1999, Quantification and immunocytochemical characteristics of trigeminal ganglion neurons projecting to the cornea: effect of corneal wounding. Eur J Pain, 3, 31-39

Ferrari, G., Bignami, F., Giacomini, C., Capitolo, E., Comi, G., Chaabane, L., Rama, P., 2014, Ocular surface injury induces inflammation in the brain: in vivo and ex vivo evidence of a corneal-trigeminal axis. Invest Ophthalmol Vis Sci, 55, 6289-6300

Garcia-Larrea, L., Peyron, R., 2013, Pain matrices and neuropathic pain matrices: a review. Pain, 154, Suppl 1:S29-43

Hirata, H., Oshinsky, M.L., 2012, Ocular dryness excites two classes of corneal afferent neurons implicated in basal tearing in rats: involvement of transient receptor potential channels. J Neurophysiol, 107, 1199-1209

Ji, R.R., Suter, M.R., 2007, p38 MAPK, microglial signaling, and neuropathic pain. Mol Pain, 3,33

Katagiri, A., Thompson, R., Rahman, M., Okamoto, K., Bereiter, D.A., 2015, Evidence for TRPA1 involvement in central neural mechanisms in a rat model of dry eye. Neuroscience, 290, 204-213

Kleinschnitz, C., Brinkhoff, J., Sommer, C., Stoll, G., 2005, Contralateral cytokine gene induction after peripheral nerve lesions: dependence on the mode of injury and NMDA receptor signaling. Brain Res Mol Brain Res, 136, 23-28

Koltzenburg, M., Wall, P.D., McMahon, S.B., 1999, Does the right side know what the left is doing? Trends Neurosci. 22, 122-127

Kurose, M., Meng, I.D., 2013, Corneal dry-responsive neurons in the spinal trigeminal nucleus respond to innocuous cooling in the rat. J Neurophysiol, 109, 2517-2522

Launay, P.S., Godefroy, D., Khabou, H., Rostene, W., Sahel, J.A., Baudouin, C., Melik Parsadaniantz, S., Reaux-Le Goazigo, A., 2015, Combined 3DISCO clearing method, 
retrograde tracer and ultramicroscopy to map corneal neurons in a whole adult mouse trigeminal ganglion. Exp Eye Res, 139, 136-143

Liang, H., Baudouin, C., Daull, P., Garrigue, J.S., Buggage, R., Brignole-Baudouin, F., 2012, In vitro and in vivo evaluation of a preservative-free cationic emulsion of latanoprost in corneal wound healing models. Cornea, 31, 1319-1329

Liang, H., Baudouin, C., Pauly, A., Brignole-Baudouin, F., 2008, Conjunctival and corneal reactions in rabbits following short- and repeated exposure to preservative-free tafluprost, commercially available latanoprost and $0.02 \%$ benzalkonium chloride. Br J Ophthalmol, 92, $1275-1282$

Lin, Z., Liu, X., Zhou, T., Wang, Y., Bai, L., He, H., Liu, Z., 2011, A mouse dry eye model induced by topical administration of benzalkonium chloride. Mol Vis, 17, 257-264

Ma, C., Shu, Y., Zheng, Z., Chen, Y., Yao, H., Greenquist, K.W., White, F.A., LaMotte, R.H., 2003, Similar electrophysiological changes in axotomized and neighboring intact dorsal root ganglion neurons. J Neurophysiol, 9, 1588-1602

Marfurt, C.F., Del Toro, D.R., 1987, Corneal sensory pathway in the rat: a horseradish peroxidase tracing study. J Comp Neurol, 261, 450-459

Marfurt, C.F., Kingsley, R.E., Echtenkamp, S.E., 1989, Sensory and sympathetic innervation of the mammalian cornea. A retrograde tracing study. Invest Ophthalmol Vis Sci, 30, 461-472 Mélik Parsadaniantz, S., Rivat, C., Rostène, W., Réaux-Le Goazigo, A., 2015, Opioid and chemokine receptor crosstalk: a promising target for pain therapy? Nat Rev Neurosci, 16, 6978.

Meng, I.D., Barton, S.T., Mecum, N.E., Kurose, M., 2015, Corneal sensitivity following lacrimal gland excision in the rat. Invest Ophthalmol Vis Sci, 56, 3347-3354 
Meng, I.D., Bereiter, D.A., 1996, Differential distribution of Fos-like immunoreactivity in the spinal trigeminal nucleus after noxious and innocuous thermal and chemical stimulation of rat cornea. Neuroscience, $72,243-254$

Mitsikostas, D.D., Sanchez del Rio, M., 2001, Receptor systems mediating c-fos expression within trigeminal nucleus caudalis in animal models of migraine. Brain Res Brain Res Rev, $35,20-35$

Muller, L.J., Vrensen, G.F., Pels, L., Cardozo, B.N., Willekens, B., 1997, Architecture of human corneal nerves. Invest Ophthalmol Vis Sci, 38, 985-994

Nakamura, A., Hayakawa, T., Kuwahara, S., Maeda, S., Tanaka, K., Seki, M., Mimura, O., 2007, Morphological and immunohistochemical characterization of the trigeminal ganglion neurons innervating the cornea and upper eyelid of the rat. J Chem Neuroanat, 34, 95-101

Patodia, S., Raivich, G., 2012, Role of transcription factors in peripheral nerve regeneration. Front Mol Neurosci, 5, 8

Rahman, M., Okamoto, K., Thompson, R., Katagiri, A., Bereiter, D.A., 2015, Sensitization of trigeminal brainstem pathways in a model for tear deficient dry eye. Pain, 156, 942-950

Rozsa, A.J., Beuerman, R.W., 1982, Density and organization of free nerve endings in the corneal epithelium of the rabbit. Pain, 14, 105-120

Sarkar, J., Chaudhary, S., Namavari, A., Ozturk, O., Chang, J.H., Yco, L., Sonawane, S., Khanolkar, V., Hallak, J., Jain, S., 2012, Corneal neurotoxicity due to topical benzalkonium chloride. Invest Ophthalmol Vis Sci, 53, 1792-1802

Shokouhi, B.N., Wong, B.Z., Siddiqui, S., Lieberman, A.R., Campbell, G., Tohyama, K., Anderson, P.N., 2010, Microglial responses around intrinsic CNS neurons are correlated with axonal regeneration. BMC Neurosci, 11, 13

Stapleton, F., Marfurt, C., Golebiowski, B., Rosenblatt, M., Bereiter, D., Begley, C., Dartt, D., Gallar, J., Belmonte, C., Hamrah, P., Willcox, M., Discomfort, T.I.W.o.C.L., 2013, The 
TFOS International Workshop on Contact Lens Discomfort: report of the subcommittee on neurobiology. Invest Ophthalmol Vis Sci, 54, TFOS 71-97

Strassman, A.M., Vos, B.P., 1993, Somatotopic and laminar organization of fos-like immunoreactivity in the medullary and upper cervical dorsal horn induced by noxious facial stimulation in the rat. J Comp Neurol, 331, 495-516

Takeda, M., Takahashi, M., Matsumoto, S., 2009, Contribution of the activation of satellite glia in sensory ganglia to pathological pain. Neurosci Biobehav Rev, 33, 784-792

Tashiro, A., Okamoto, K., Chang, Z., Bereiter, D.A., 2010, Behavioral and neurophysiological correlates of nociception in an animal model of photokeratitis. Neuroscience, $169,455-462$

Xiong, C., Chen, D., Liu, J., Liu, B., Li, N., Zhou, Y., Liang, X., Ma, P., Ye, C., Ge, J., Wang, Z., 2008, A rabbit dry eye model induced by topical medication of a preservative benzalkonium chloride. Invest Ophthalmol Vis Sci, 49, 1850-1856 

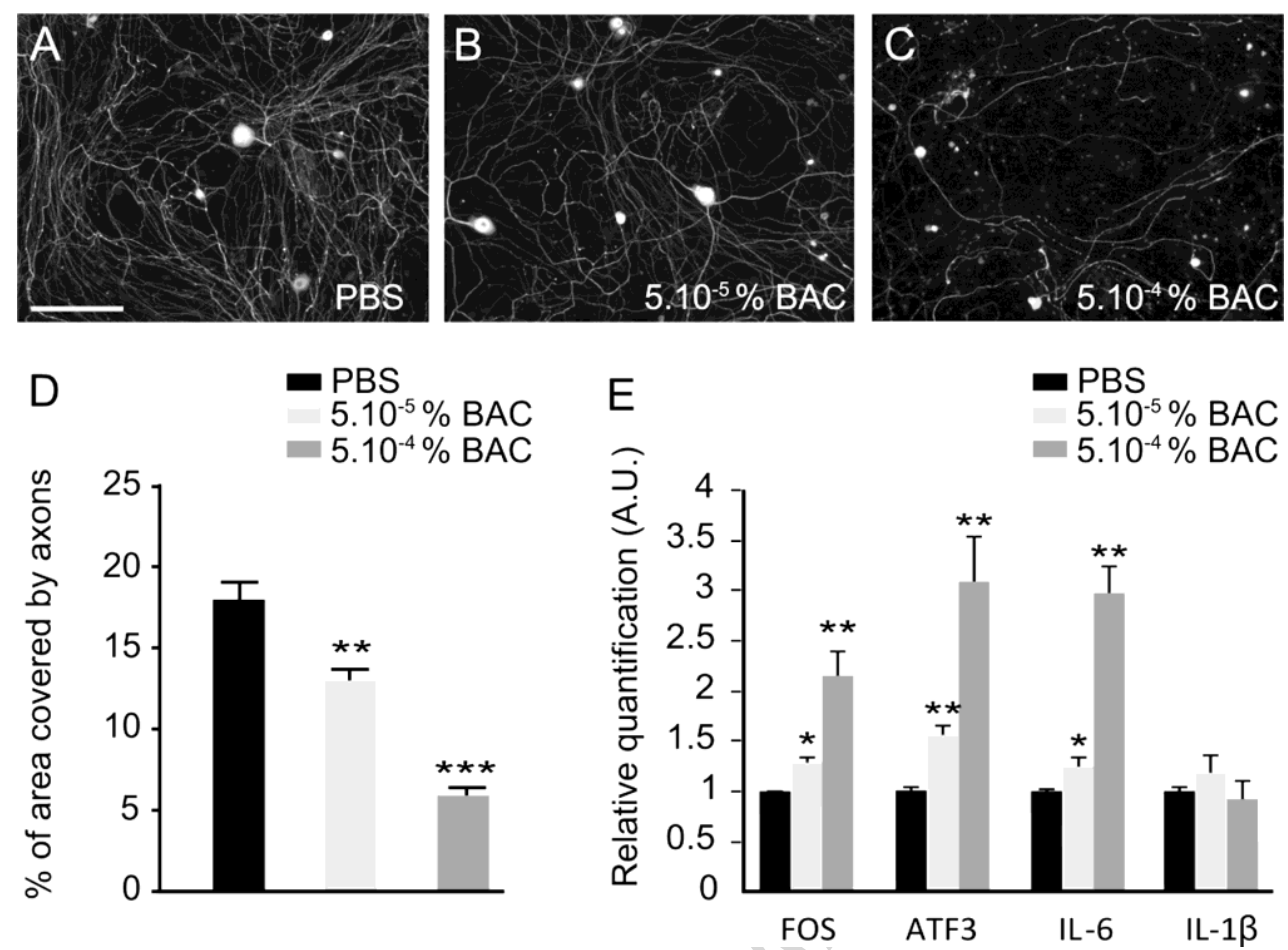

Fig. 1 
A
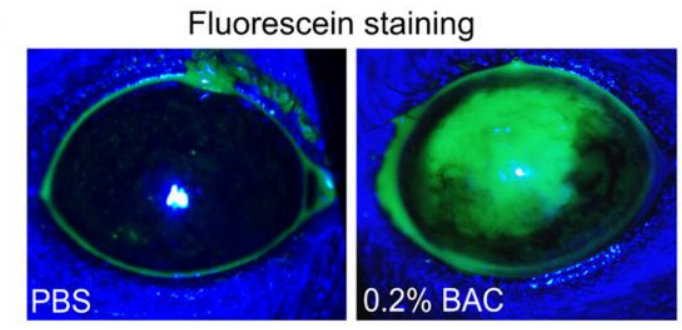

B

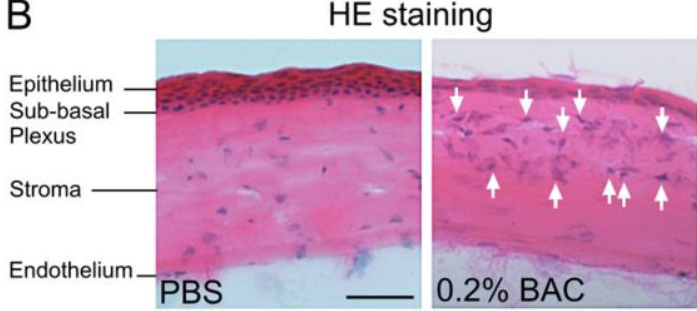

C

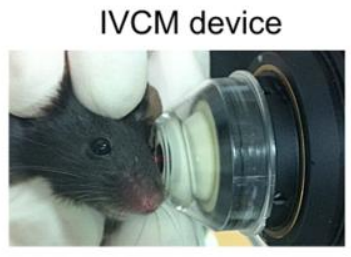

E

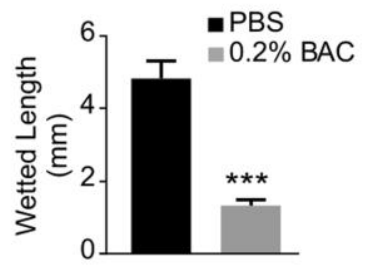

D Epithelium
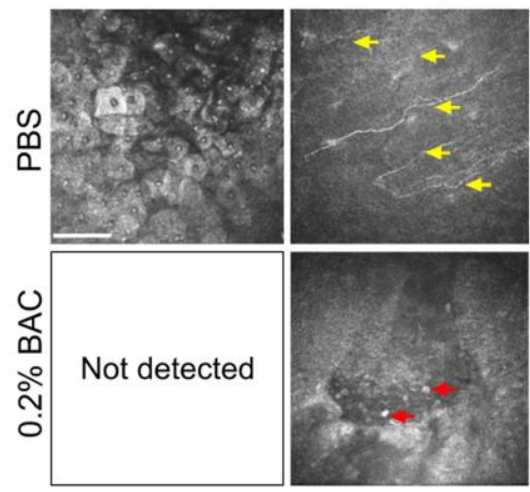

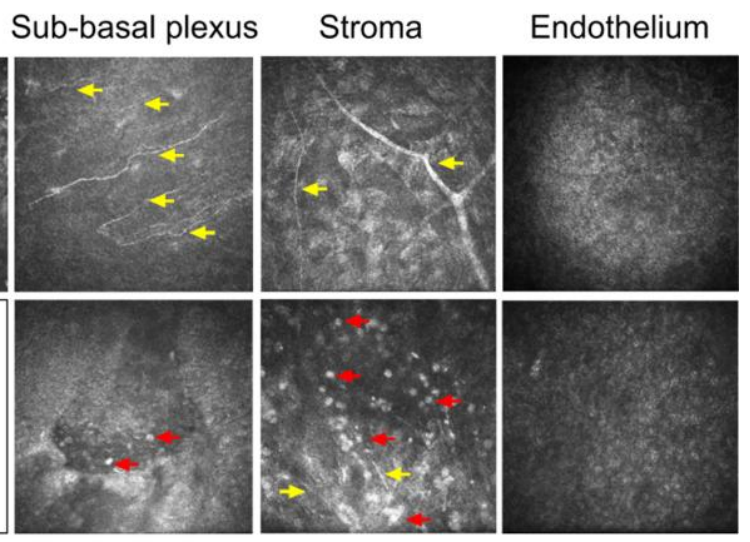

Endothelium

F
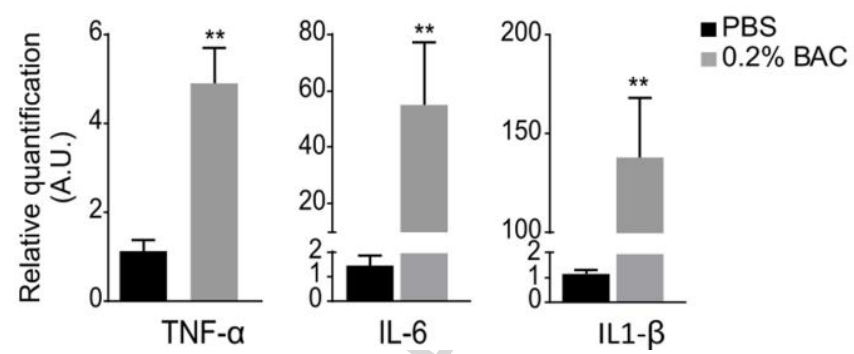

Fig. 2 
A
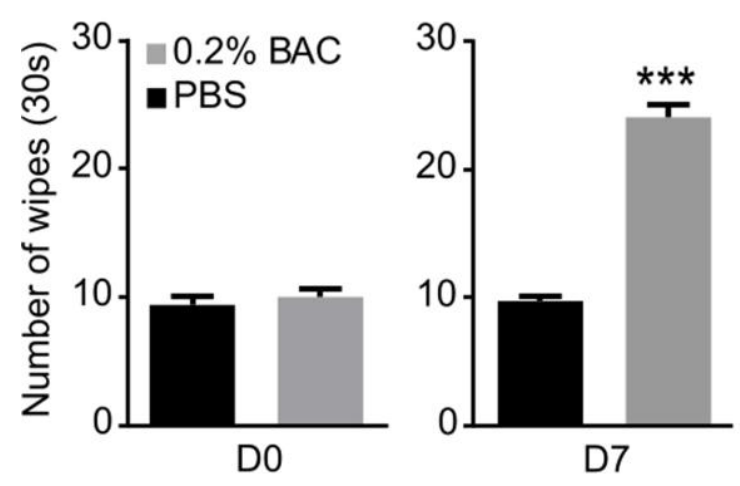

Fig. 3
B

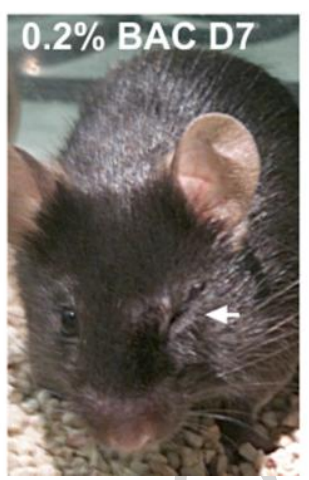

C

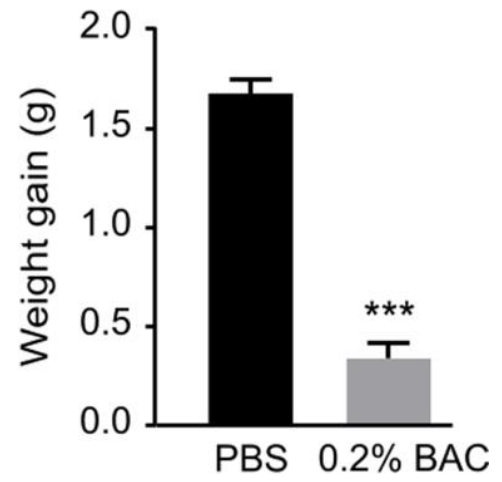




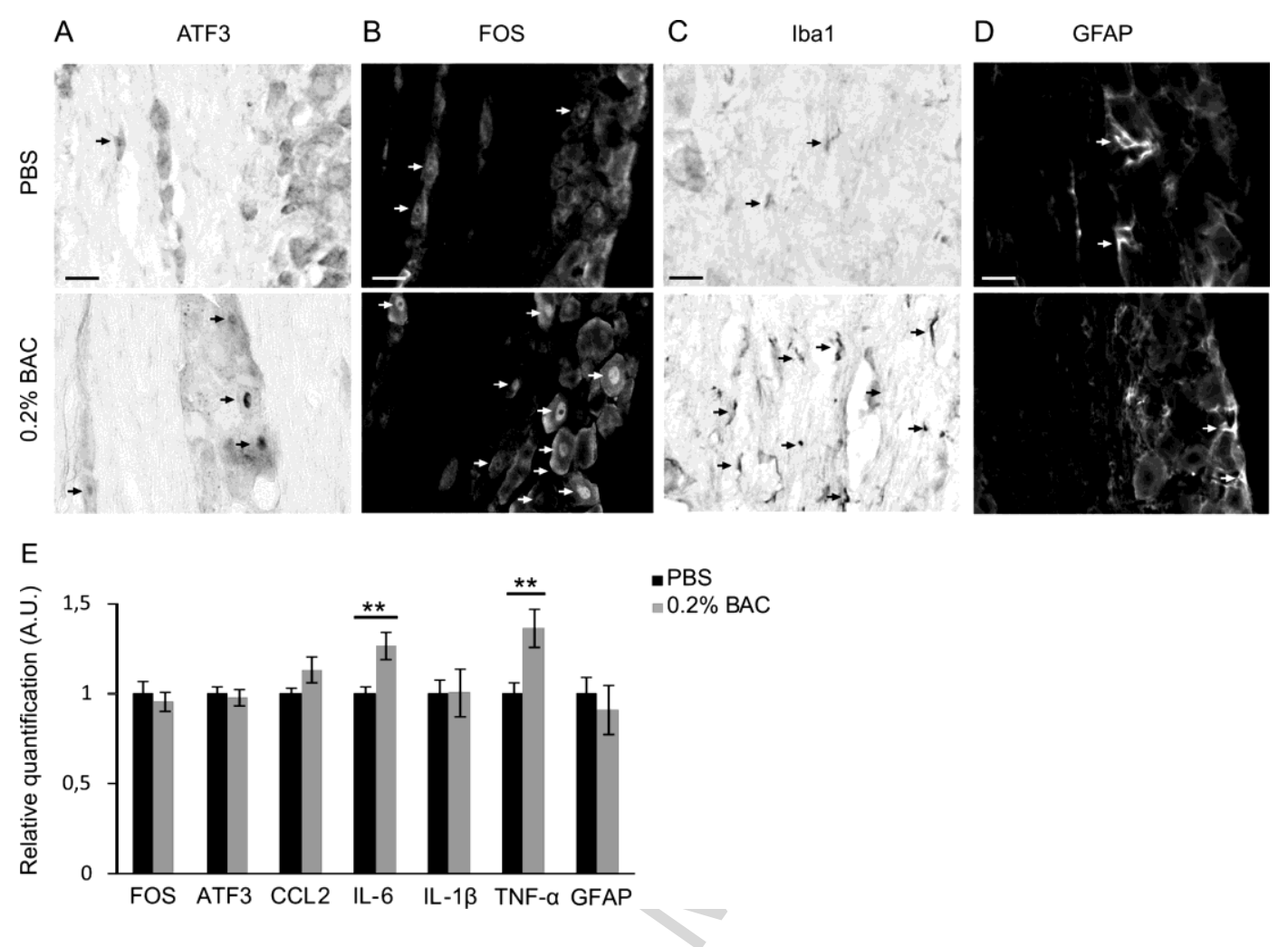

Fig. 4 
Trigeminal subnucleus $\mathrm{Vi} / \mathrm{Vc}$ region

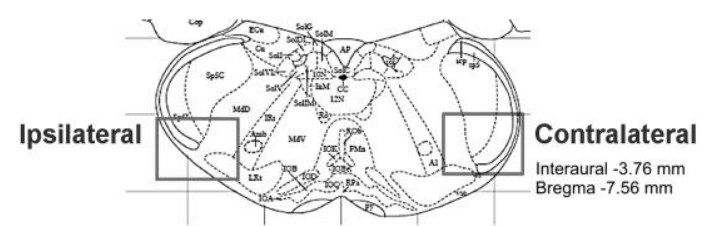

A

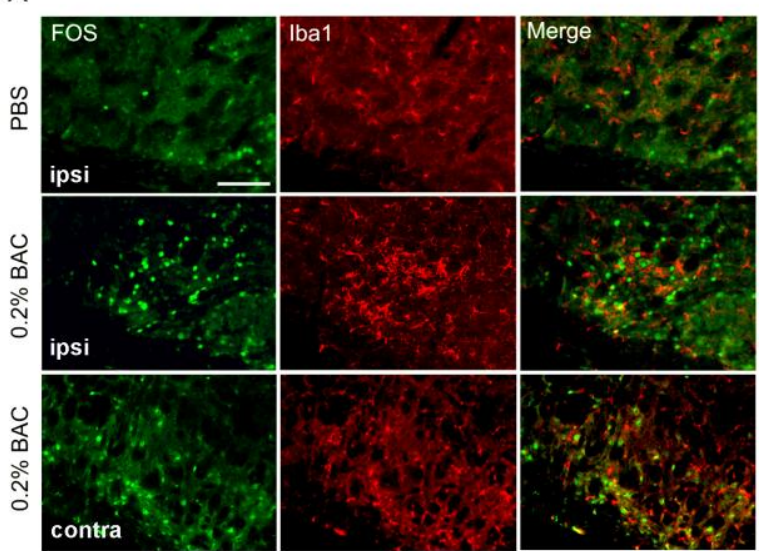

B

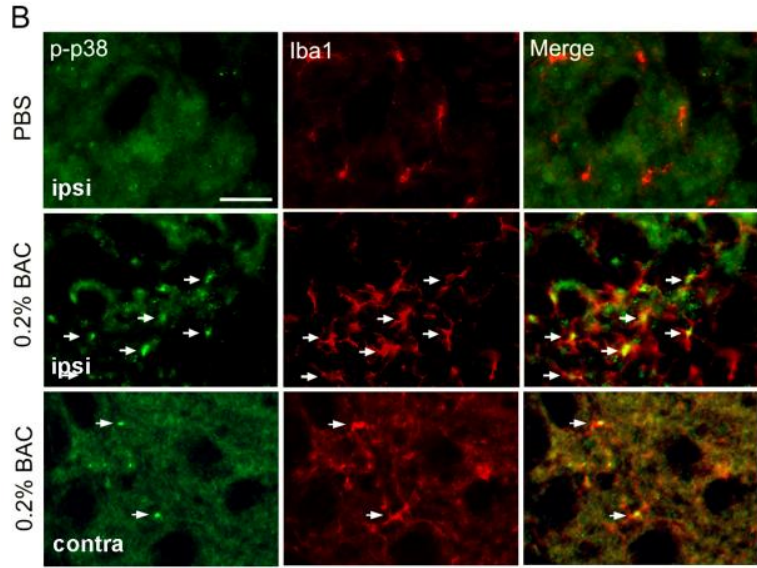

Trigeminal subnucleus $\mathrm{Vc} / \mathrm{C} 1$ region

Ipsilateral

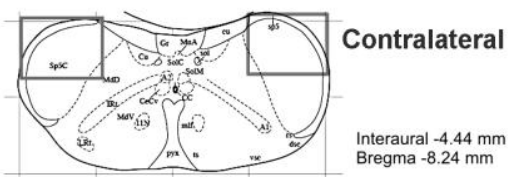

C

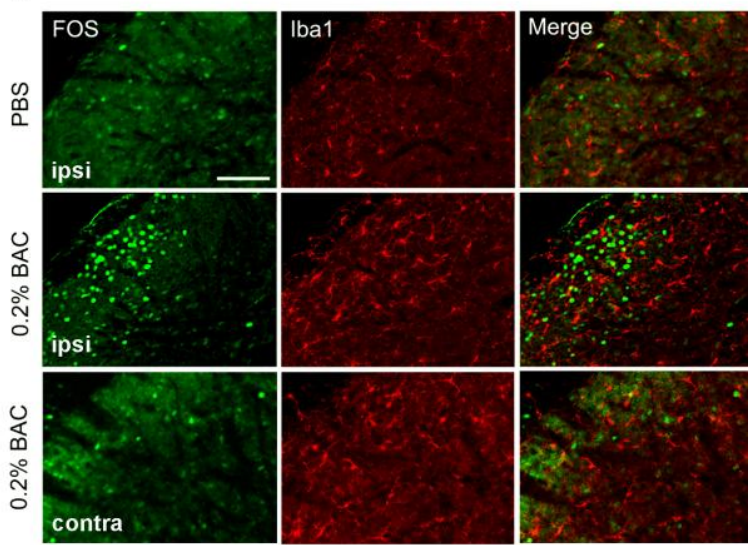

D

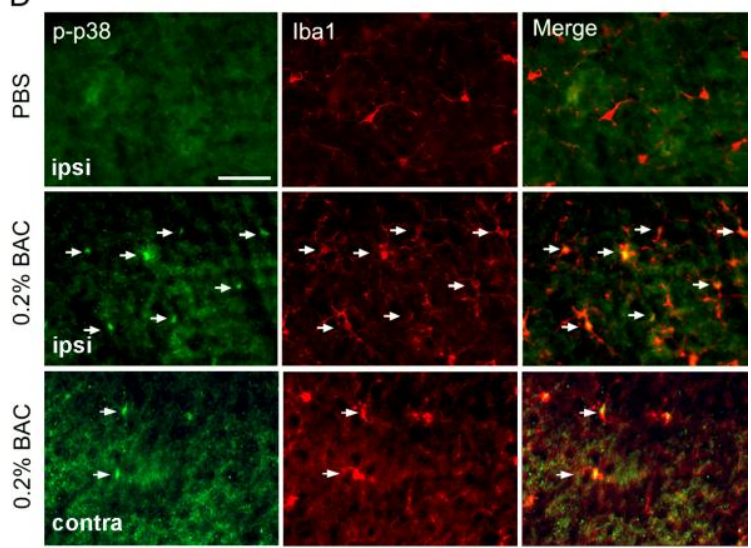

Fig. 5 
Facial Motor Nucleus

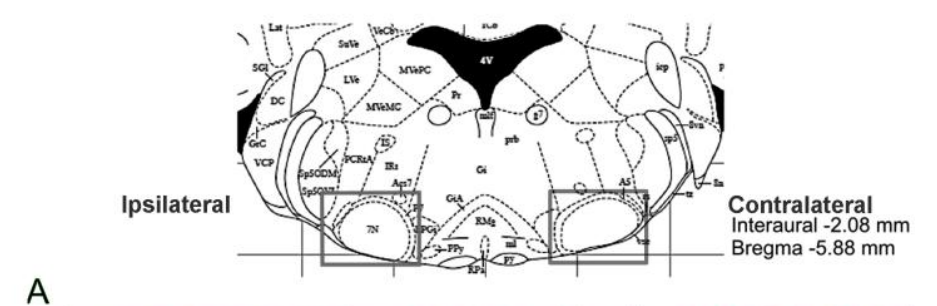

A
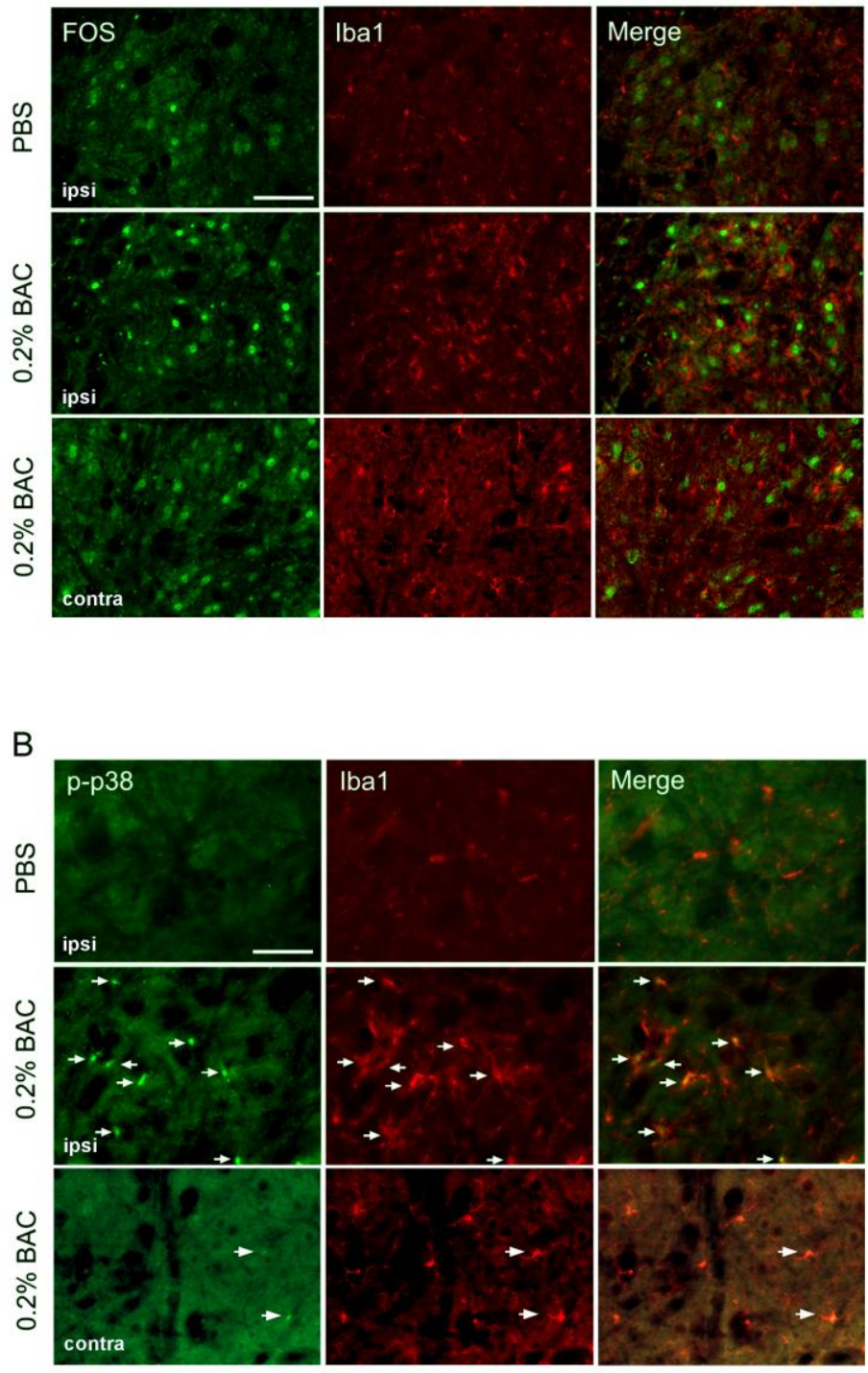

Fig. 6 


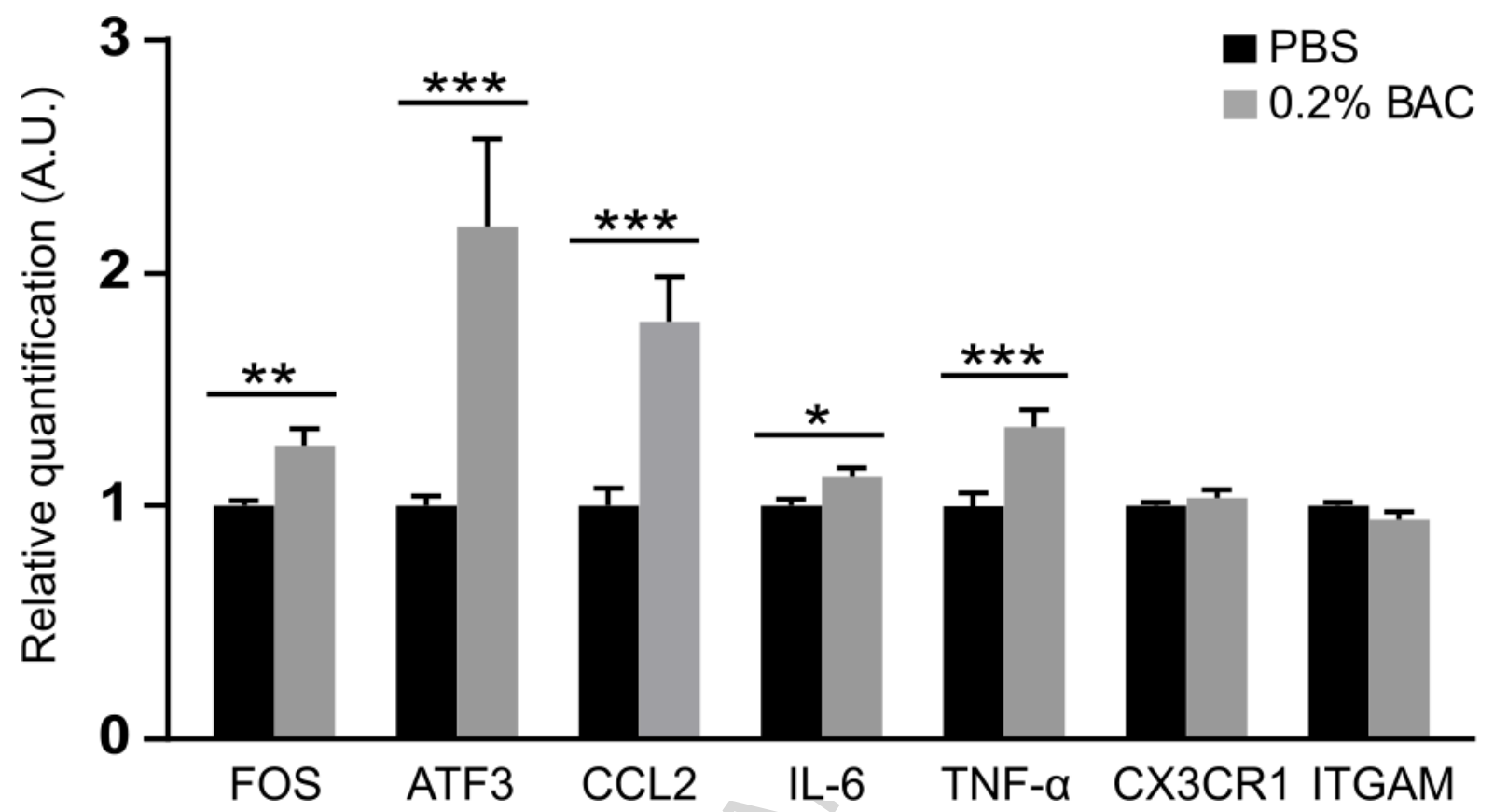

Fig. 7 

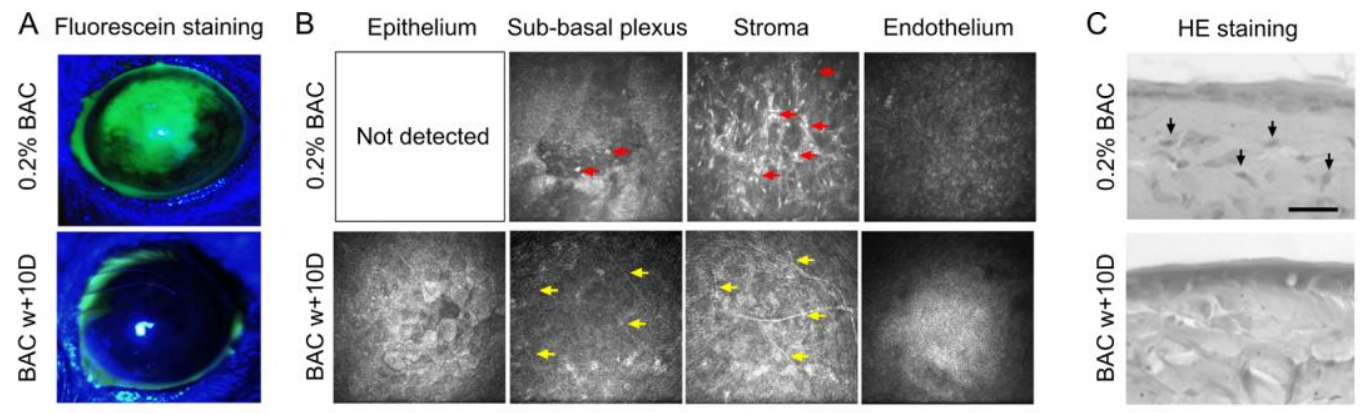

D
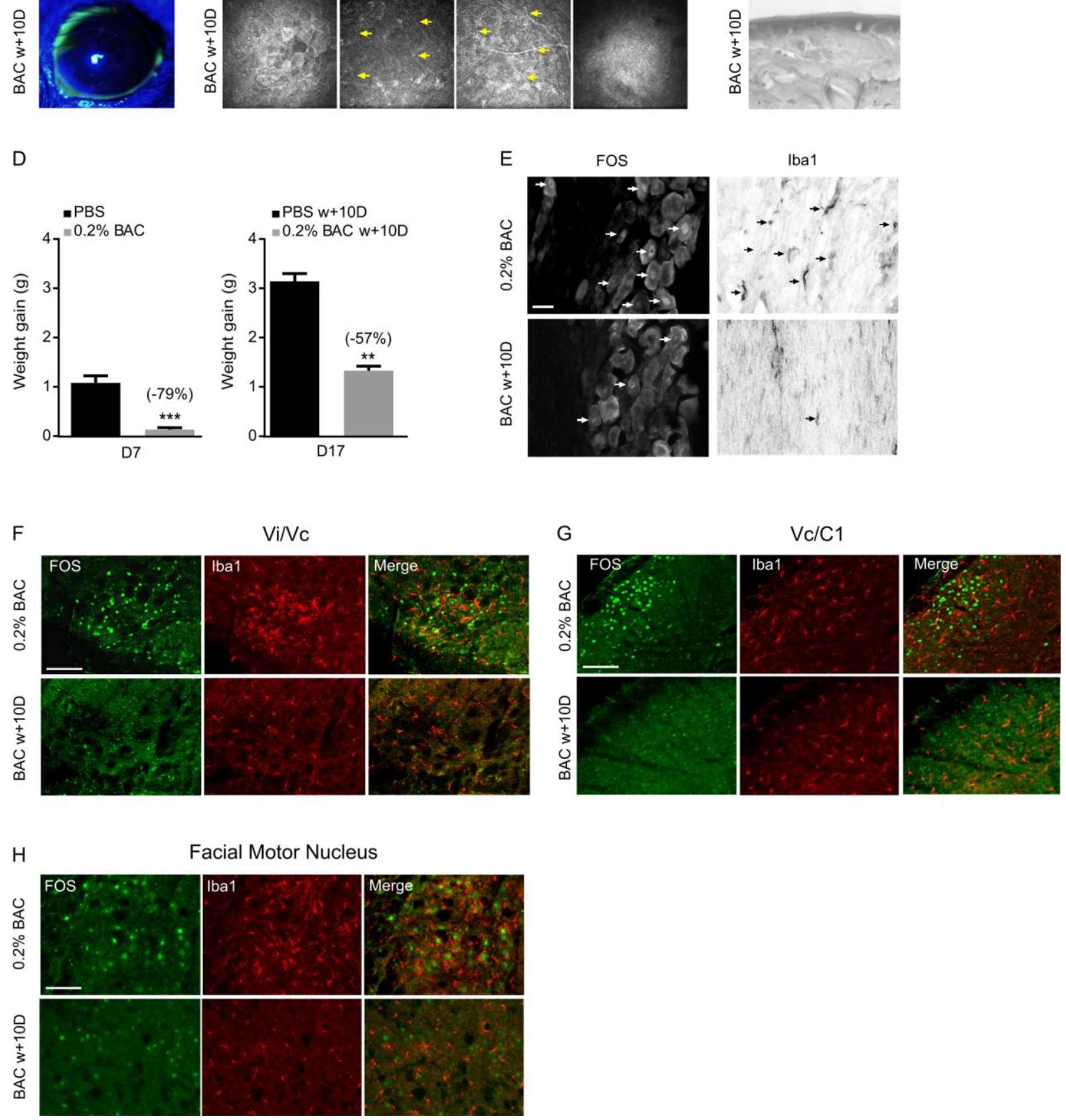

Fig. 8 


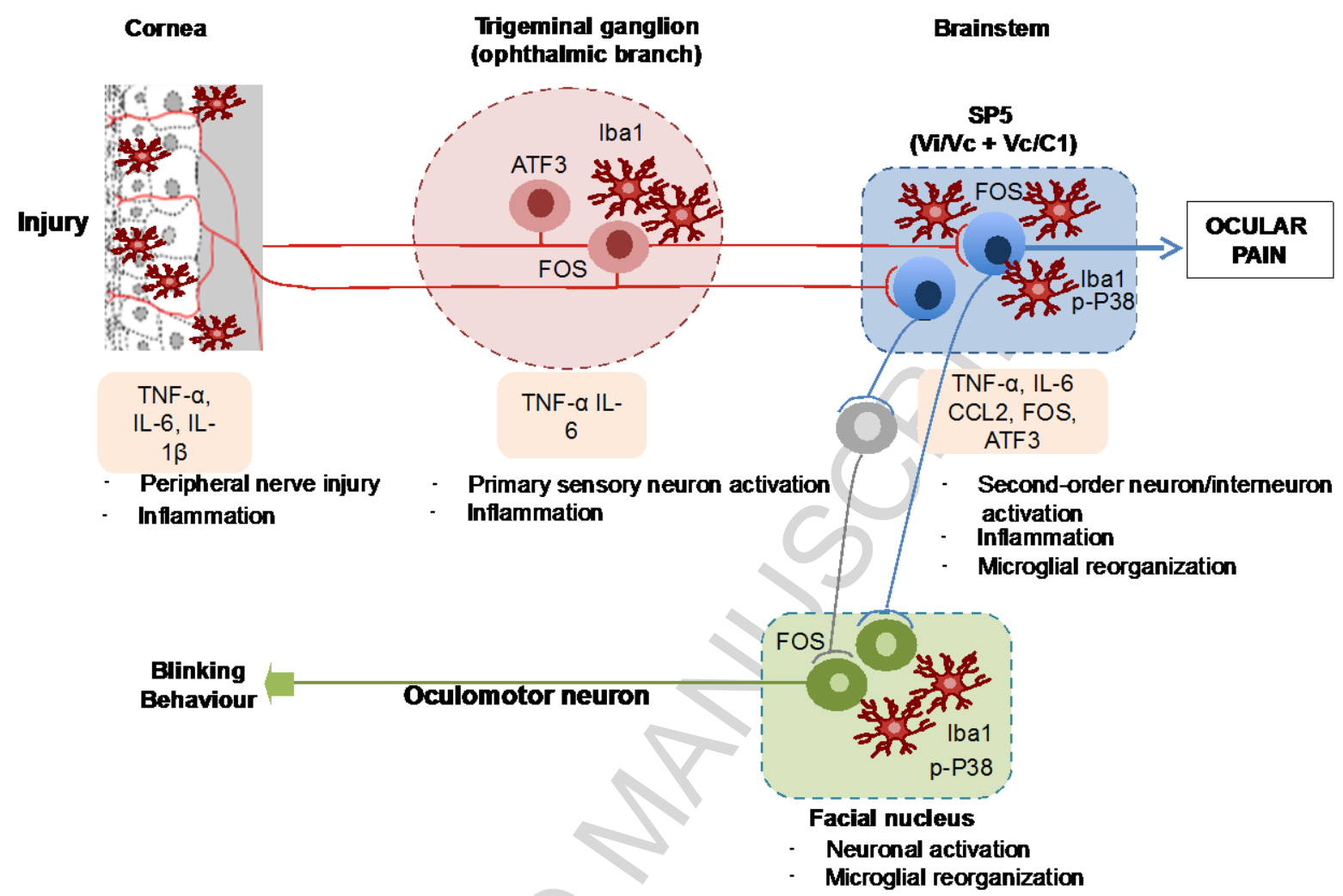

Figure 9

Graphical abstract

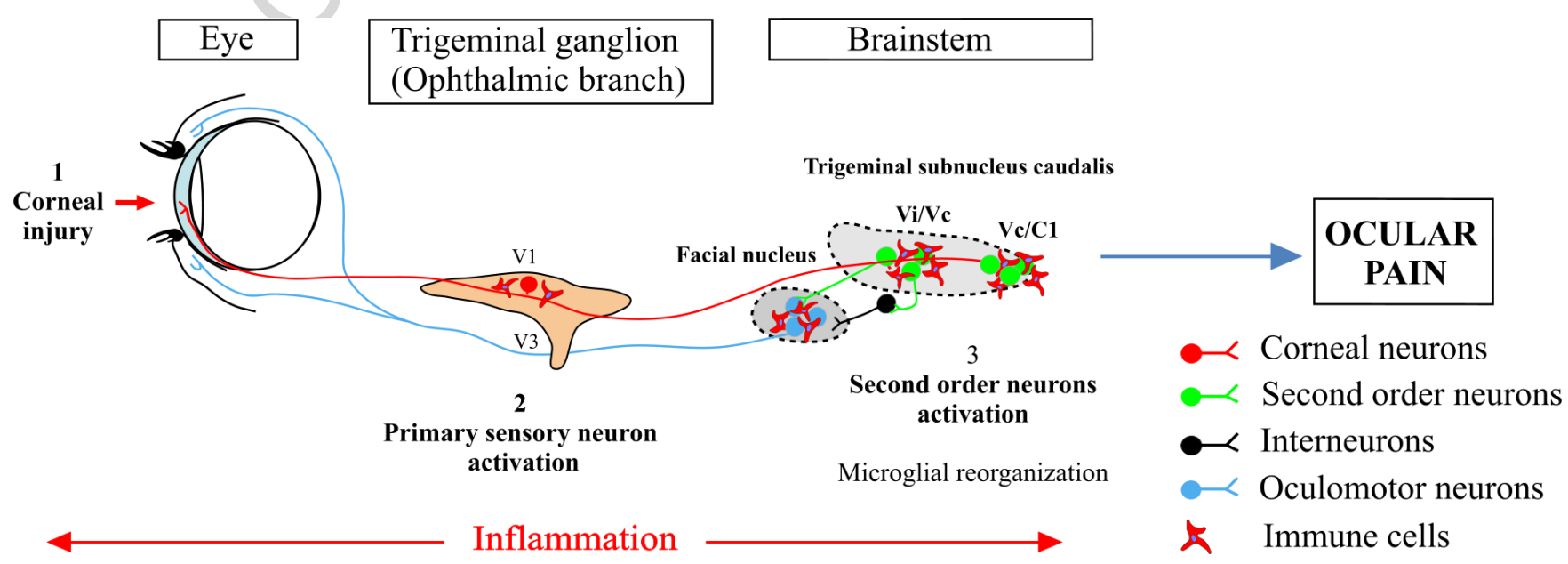

